\title{
Global analysis of charge exchange meson production at high energies
}

\author{
J. Nys, ${ }^{1,2,3,}$ A. N. Hiller Blin, ${ }^{4, \dagger}$ V. Mathieu, ${ }^{5}$ C. Fernández-Ramírez, ${ }^{6}$ A. Jackura, ${ }^{2,3}$ A. Pilloni, ${ }^{5}$ \\ J. Ryckebusch, ${ }^{1}$ A. P. Szczepaniak, ${ }^{2,3,5}$ and G. Fox ${ }^{7}$ \\ (Joint Physics Analysis Center) \\ ${ }^{1}$ Department of Physics and Astronomy, Ghent University, Ghent 9000, Belgium \\ ${ }^{2}$ Center for Exploration of Energy and Matter, Indiana University, Bloomington, Indiana 47403, USA \\ ${ }^{3}$ Physics Department, Indiana University, Bloomington, Indiana 47405, USA \\ ${ }^{4}$ Institut für Kernphysik \& PRISMA Cluster of Excellence, Johannes Gutenberg Universität, \\ D-55099 Mainz, Germany \\ ${ }^{5}$ Theory Center, Thomas Jefferson National Accelerator Facility, \\ 12000 Jefferson Avenue, Newport News, Virginia 23606, USA \\ ${ }^{6}$ Instituto de Ciencias Nucleares, Universidad Nacional Autónoma de México, \\ Ciudad de México 04510, Mexico \\ ${ }^{7}$ School of Informatics and Computing, Indiana University, Bloomington, Indiana 47405, USA
}

(Received 17 June 2018; published 21 August 2018)

\begin{abstract}
Many experiments that are conducted to study the hadron spectrum rely on peripheral resonance production. Hereby, the rapidity gap allows the process to be viewed as an independent fragmentation of the beam and the target, with the beam fragmentation dominated by production and decays of meson resonances. We test this separation by determining the kinematic regimes that are dominated by factorizable contributions, indicating the most favorable regions to perform this kind of experiments. In doing so, we use a Regge model to analyze the available world data of charge exchange meson production with beam momentum above $5 \mathrm{GeV}$ in the laboratory frame that are not dominated by either pion or Pomeron exchanges. We determine the Regge residues and point out the kinematic regimes which are dominated by factorizable contributions.
\end{abstract}

DOI: 10.1103/PhysRevD.98.034020

\section{INTRODUCTION}

The new generation of high statistics experiments e.g., Belle II, BESIII, CLAS12, CMS, COMPASS, GlueX, J-PARC, LHCb, and PANDA, have dedicated programs to study the hadron spectrum, whose quantitative description is pivotal for a complete understanding of quantum chromodynamics (QCD). These experiments demand a high level of precision in the amplitude analysis [1] necessary to obtain reliable extractions of hadron properties from the data. In particular, the diffraction of photons or mesons on the nucleon target at high energies, as studied at GlueX, CLAS12, and COMPASS, is expected to provide information on hybrids, exotics, and the gluonic degrees of

\footnotetext{
*jannes.nys@ugent.be
}

hillerbl@uni-mainz.de

Published by the American Physical Society under the terms of the Creative Commons Attribution 4.0 International license. Further distribution of this work must maintain attribution to the author(s) and the published article's title, journal citation, and DOI. Funded by SCOAP . freedom, via independent fragmentation of the beam and of the target (see Fig. 1), with the beam fragmentation dominated by production and decays of mesons.

Regge phenomenology underlies such processes and provides the theoretical framework for studying high energy scattering. Other high-energy pQCD frameworks used in exclusive reactions, such as GPDs, meson DAs, ..., require a hard interaction scale for factorization to hold [2]. Regge theory, on the other hand, is applicable for large

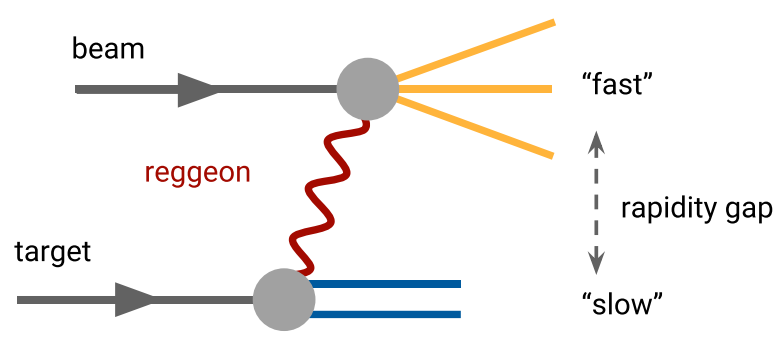

FIG. 1. Illustration of the factorization of peripheral meson production. Diffractive events are typically characterized by a gap in the rapidity distribution of the produced particles. 
center-of-mass energies $(s)$, forward scattering angles (small $-t$ ), small meson masses, and, most importantly, the lack of a hard scale. In Regge theory, resonances in the exchanged channel are related to each other and are described by the Regge trajectories, also referred to as reggeons. Specifically, the pion diffractive dissociation at COMPASS is dominated by exchanges of reggeons with vacuum quantum numbers, including the Pomeron $(\mathbb{P})$. Photon induced reactions at the Jefferson Lab (JLab) may also proceed by exchange of reggeons with nonvacuum quantum numbers. Regge trajectories provide specific information about the dynamics responsible for the formation of resonances [3-5] that can be used to constrain amplitudes of other reactions, e.g., production of light hadrons in heavy flavor hadron decays. In order to confidently separate the beam and target fragmentation in peripheral scattering, it is necessary to establish the validity of Regge pole factorization. Establishing the production mechanism in the production of resonances is also a necessary first step in the determination of their quantum numbers and other characteristics, as their quark model nature [6]. This is particularly relevant when searching for new states, e.g., hybrid mesons, which is one of the main goals of the spectroscopy program at JLab $[7,8]$.

In the near future, new data on peripheral resonance production will be coming primarily from JLab experiments, and therefore, it is important to validate Regge mechanisms for beam energies of $E_{\gamma} \sim O(10 \mathrm{GeV})$. Even though high energy peripheral processes are expected to be dominated by exchanges of leading Regge poles, there are subleading singularities, e.g., Regge cuts and/or poles in daughter trajectories, which have to be assessed [9]. With this goal in mind, we have recently studied $\pi N$ scattering and $\pi^{0} N, \eta N, \pi \Delta$, and neutral vector meson photoproduction [10-16], obtaining several results that we briefly summarize below. In $\pi^{0}$ photoproduction, we used finite energy sum rules (FESR's) [14] to demonstrate that there is a good agreement between the partial wave models (PWA) for low energy amplitudes [17-24] and the Regge parametrization of the high-energy data. In $\eta$ photoproduction, the PWA models are less constrained by the available lowenergy data, and we have shown how the high-energy data can help reduce uncertainties, specifically those related to unnatural exchanges [12,14]. Our prediction for the $\pi^{0}$ photoproduction cross section [11] compares favorably with the CLAS data [25], and our results on $\pi^{0}, \eta$, and $\eta^{\prime}$ photoproduction beam asymmetries $[15,16]$ are in agreement with the GlueX results [7,26]. The main conclusion one can draw from these comparisons is that, at forward scattering angles, the natural Regge poles dominate over the unnatural ones and over the nonpole contributions. In general, for natural exchanges we have found a good agreement with factorization. Specifically, a zero in the residue of the $\omega$ exchange in $\pi N$ [10] implies a similar behavior for the photoproduction reactions with $\omega$ exchange. Indeed, a zero is found in the $\eta$ photoproduction amplitude, and a strong dip is present in the cross section for $\pi^{0}$ photoproduction [12]. Complementary to these analyses, we now study reactions with meson beams sharing the same nucleon residues. For these reactions, the amount of high-energy data is abundant, which allows for a detailed study of both the residue factorizability and the energy dependence of the observables. Additionally, mesonic beams allow for less exchanges compared to photon beams, therefore allowing us to study the dominant natural exchanges in isolations. For those kinematics where Regge factorization holds, information about the residues can be applied to photoproduction reactions.

Since the Regge picture has been well established for $\pi N$ scattering and $\pi$ and $\eta^{(/)}$photoproduction off the proton, in this work, we proceed to examine the Regge pole model in a global analysis of several quasi-two-body reactions of interest to peripheral resonance production. When subleading contributions, such as Regge cuts or daughters, are accounted for in the amplitudes, the factorization approximation is violated. We aim to identify the kinematics for which such violations can be expected, while for processes dominated by factorizable exchanges we provide amplitudes and residues that are compatible with the world data at high energies. These can be used to model the production mechanism in fragmentation experiments, allowing the isolation of the resonant part intended to search for hybrids.

In the Regge pole approximation, the amplitudes are well constrained by unitarity and analyticity and are specified by a small number of parameters. Thus, in principle, a large enough data set in principle makes it possible to test the Regge pole dominance hypothesis [27]. In this work, we perform a global analysis of all available data on charge exchange (CEX) quasi-two-body reactions with meson beams that are dominated by vector and tensor Regge trajectories. Except for the Pomeron exchange, which does not contribute to CEX reactions, vector and tensor exchanges are expected to dominate in the energy range of interest. Furthermore, we exclude processes in which pion exchange is possible. At high energies, the pion pole is close to the physical region and becomes more sensitive to the subleading Regge contributions. This, in general, requires special treatment $[13,28,29]$. The data set considered in this paper includes 23 reactions and 1271 differential cross section data points, as described in Sec. III B, and summarized in Table II.

The paper is organized as follows. We discuss the main features of the formalism in Sec. II and Sec. III A, leaving the technicalities to the Appendices. The results of the fits are discussed in Sec. III B, and in Sec. V, we summarize the main conclusions. The kinematics and our conventions are discussed in detail in Appendix A. Appendix B contains a summary of the effect of factorization of the Regge residues on the forward behavior of the helicity amplitudes. The interaction Lagrangians used in the fits are contained in Appendix C, with estimates of the corresponding coupling 
constants derived in Appendix D. Our method for building Regge amplitudes from single-particle exchange amplitudes is discussed with an example in Appendix E. Finally, Appendix $\mathrm{F}$ provides the expressions that allow one to determine the $t$-channel helicity residues directly from the $s$-channel residues and vice versa, without the need for introducing Lagrangians.

\section{FORMALISM}

We consider reactions of the type (see Fig. 2)

$$
1\left(p_{1}, \mu_{1}\right)+2\left(p_{2}, \mu_{2}\right) \rightarrow 3\left(p_{3}, \mu_{3}\right)+4\left(p_{4}, \mu_{4}\right),
$$

where the $p_{i}$ 's are the 4-momenta, and the $\mu_{i}$ 's are the helicities in the center of mass frame, referred to as the $s$-channel frame. The standard Mandelstam variables are $s=\left(p_{1}+p_{2}\right)^{2}, t=\left(p_{1}-p_{3}\right)^{2}$, and $u=\left(p_{1}-p_{4}\right)^{2}$. We parametrize the high-energy $s$-channel helicity amplitudes following the analysis of Cohen-Tannoudji et al. [30], which leads to a factorized form as discussed in [27]. Specifically, in the large $s$ limit the amplitude of a Regge pole $(e)$ described by a trajectory $\alpha_{e}(t)$, which in the $s$-channel physical region is approximated by a linear function, $\alpha_{e}(t)=\alpha_{e}^{0}+\alpha_{e}^{1} t$, is given by

$$
\begin{aligned}
A_{\mu_{4} \mu_{3} \mu_{2} \mu_{1}}(s, t)= & -\frac{\pi \alpha_{e}^{1}}{2} \beta_{\mu_{4} \mu_{3} \mu_{2} \mu_{1}}^{e}(t) \\
& \times \frac{\zeta_{e}+e^{-i \pi \alpha_{e}(t)}}{\sin \pi \alpha_{e}(t)}\left(\frac{s}{s_{0}}\right)^{\alpha_{e}(t)},
\end{aligned}
$$

where $\zeta_{e}= \pm 1$ is the reggeon signature, and $s_{0}$ is a scale above which the pole approximation is expected to dominate, $s \gtrsim s_{0}$. The latter is related to the range of the strong interaction. In the following, we use $s_{0}=1 \mathrm{GeV}^{2}$. Notice that fixing $s_{0}$ is a convention. Changing the value of $s_{0}$ introduces solely a different $t$-dependence, which we absorb into the residue. We refer to right (wrong) signature points as those kinematics for which the signature factor $\zeta_{e}+e^{-i \pi \alpha_{e}(t)}$ in Eq. (2) is finite (vanishes). Except for kinematic factors, discussed below, Regge theory does not fix the $t$-dependence of the residues $\beta_{\mu_{4} \mu_{3} \mu_{2} \mu_{1}}^{e}(t)$. Unitarity in the $t$-channel requires that the trajectory and residues are real functions in the kinematic region of interest and that the $t$-channel helicity residues are factorizable in a separate
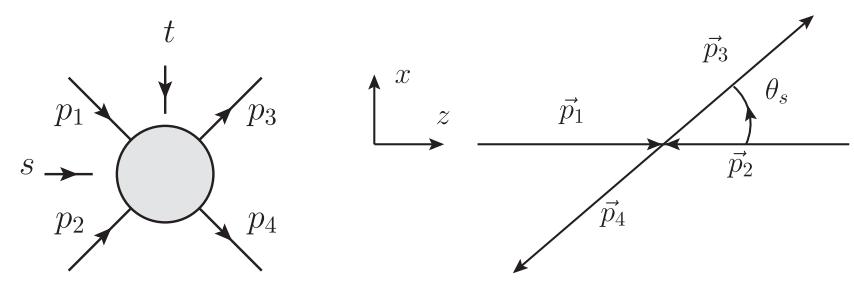

FIG. 2. Kinematics for the reaction $1+2 \rightarrow 3+4$ in the $s$-channel center of the mass frame. contribution coming from the meson vertex and a contribution from the baryon vertex. It was shown in [27] that in the high-energy limit, due to properties of the crossing matrix, the $s$-channel helicity residues must also be factorizable. Hence, the residues $\beta_{\mu_{4} \mu_{3} \mu_{2} \mu_{1}}^{e}(t)$ in Eq. (2) can be written in a product form [31]

$$
\beta_{\mu_{4} \mu_{3} \mu_{2} \mu_{1}}^{e}(t)=\beta_{\mu_{2} \mu_{4}}^{e 24}(t) \beta_{\mu_{1} \mu_{3}}^{e 13}(t) .
$$

In the following, the $\beta_{\mu_{2} \mu_{4}}^{e 24}(t)$ and $\beta_{\mu_{1} \mu_{3}}^{e 13}(t)$ will be referred to as the bottom and top or meson and nucleon residues, respectively. The superscripts indicate which external particles the residue depends upon. This factorized form illustrates the role played by coupled channels since the residues $\beta_{\mu_{i} \mu_{j}}^{e i j}(t)$ in Eq. (3) (where $(i, j)=(1,3)$ or $(i, j)=(2,4))$ are shared among various reactions which involve the same particles.

The function $\sin \pi \alpha_{e}(t)$ in the denominator of Eq. (2) reflects the existence of particle poles in the $t$-channel with integer spins $J$ given by the value of the trajectory at a pole, $J=\alpha_{e}\left(t=m_{e}^{2}\right)$. Regge amplitudes involve particles of definite parity and naturality $\eta=P(-1)^{J}= \pm 1$, which is accounted for by the signature factor $\left(\zeta_{e}+e^{-i \pi \alpha_{e}(t)}\right) / 2$. For physical spins, it reduces to $\left(\zeta_{e}+(-1)^{J}\right) / 2$ and retains only the poles which satisfy $\eta P=\zeta_{e}$. Since poles with negative spins are unphysical, the residues must contain additional zeros. For example, zeros in even (odd) signature residues must be present at values of $t$ for which $\alpha_{e}(t)$ is equal to an even (odd) negative integer. In addition, $\alpha_{e}=0$ in the even signature trajectory may occur at a value of $t$ in the $s$-channel physical region. For some trajectories, this would correspond to an unphysical exchange of a ghost spin- 0 particle. This happens in the $a_{2}$ trajectory, ${ }^{1}$ where $\alpha_{a_{2}}(t)=0$ corresponds to negative values of $t$, and therefore, an additional zero must be included at $\alpha_{a_{2}}(t)=0$ in the residue of the $a_{2}$ exchange. Hadron Regge trajectories are observed to satisfy the so-called exchange degeneracy (EXD), which can be understood in terms of local Reggeresonance duality and large- $N_{c}$ limit [6,32]. As a consequence, residues of the $a_{2}$ and $\rho$ exchanges should have the same $t$-dependence. ${ }^{2}$ Together with the requirement for zeros at the negative values of $\alpha_{e}$ with the right signature, the residues of $a_{2}$ and $\rho$ must contain zeros at all nonpositive spins. While the above reasoning invokes EXD, which holds only approximately, the zeros at $\alpha_{e}(t)=0$ are more general, as will be discussed at the end of this section. Zeros at non-negative opposite signature spins, such as the

\footnotetext{
${ }^{1}$ We denote reggeons by their lowest spin particles. Also, $K^{*} \equiv K^{*}(892)$ and $K_{2}^{*} \equiv K_{2}^{*}(1430)$.

${ }^{2}$ Two types of EXD can be distinguished: weak EXD, which requires the trajectories of opposite signature exchanges to be equal, and strong EXD, which additionally requires the residues to be equal. We will use the term EXD interchangeably for both cases, since the type of EXD will be clear from the context.
} 
zero at $\alpha_{\rho}(t)=0$, are referred to as wrong signature zeros (WSZ). To include all the expected zeros, as discussed above, we require $[9,33,34]$

$$
\beta_{\mu_{4} \mu_{3} \mu_{2} \mu_{1}}^{e}(t) \propto \frac{1}{\Gamma\left(\alpha_{e}(t)-l_{e}+1\right)},
$$

where $l_{e}$ is the lowest physical spin on the trajectory $\alpha_{e}(t)$ or on the trajectory of its exchange-degenerate partner, i.e., $l_{\rho}=l_{a_{2}}=1$. Since it is not known a priori which one of the two factorizable residues the zeros should be attributed to, we pull this factor out of the product of residues and absorb it in the definition of the remaining terms in Eq. (2). The trajectories are assumed to be linear functions and are given in Table I. Notice that we assume weak degeneracy for all exchanges: $\alpha_{\rho}(t)=\alpha_{a_{2}}(t)$ and $\alpha_{K^{*}}(t)=\alpha_{K_{2}^{*}}(t)$.

As discussed in Appendix B, for $t \rightarrow 0$ the most singular behavior of residues corresponding to $t$-channel exchanges of definite parity is given by $\beta_{\mu_{i} \mu_{j}}^{e i j}(t) \sim \sqrt{-t}\left|\mu_{i}-\mu_{j}\right|$. In order to make this kinematic $t$-dependence explicit, we define the reduced residues $\hat{\beta}_{\mu_{i} \mu_{j}}(t)$,

$$
\beta_{\mu_{i} \mu_{j}}^{e i j}(t)=\sqrt{-t}\left|\mu_{i}-\mu_{j}\right| \hat{\beta}_{\mu_{i} \mu_{j}}^{e i j}(t),
$$

which are regular in $t$. The general form of the large $s$ amplitude implied by Regge theory is therefore given by

$$
\begin{aligned}
A_{\mu_{4} \mu_{3} \mu_{2} \mu_{1}}\left(s \gg s_{0}, t\right)= & \sqrt{-t}\left|\mu_{1}-\mu_{3}\right| \sqrt{-t}^{\left|\mu_{2}-\mu_{4}\right|} \\
& \times \hat{\beta}_{\mu_{1} \mu_{3}}^{e 13}(t) \hat{\beta}_{\mu_{2} \mu_{4}}^{e 24}(t) \mathcal{F}_{e}(s, t),
\end{aligned}
$$

where $\mathcal{F}_{e}(s, t)$ is defined below. In the phenomenological analysis of the data, we have found that one obtains better fits if the $t$-dependence implied by the exact angular behavior of the amplitude is used instead of its limit corresponding to the high-energy scattering in the forward direction, $z_{s} \rightarrow 1+2 t^{\prime} / s$, where $z_{s}$ is the cosine of the scattering angle in the $s$-channel frame, and $t^{\prime}=t-t_{\text {min }}$ with $t_{\min }=t\left(z_{s}=+1\right)=O(1 / s)$ [34]. Specifically, from the overall angular-momentum conservation it follows that the $s$-channel helicity amplitude is proportional to the half-angle factor $[35,36]$

TABLE I. Considered exchanges with their relevant quantum numbers and corresponding trajectories used as input for the global SU(3)-EXD fit $(\alpha(1))$ and as obtained from the unconstrained fit $(\alpha(2))$. The Mandelstam variable $t$ must be expressed in units $\mathrm{GeV}^{2}$.

\begin{tabular}{llccc}
\hline \hline Reggeon & $I^{G \zeta \eta}$ & $l_{e}$ & $\alpha(1)$ & $\alpha(2)$ \\
\hline$\rho$ & $1^{+-+}$ & 1 & $0.5+0.9 t$ & $0.51+0.82 t$ \\
$a_{2}$ & $1^{-++}$ & 1 & $0.5+0.9 t$ & $0.42+0.90 t$ \\
$K^{*}$ & $1^{-+}$ & 1 & $0.35+0.9 t$ & $0.35+0.9 t$ \\
$K_{2}^{*}$ & $1^{++}$ & 1 & $0.35+0.9 t$ & $0.35+0.9 t$ \\
$b_{1}$ & $1^{+--}$ & 0 & $\cdot$ & $0.7 t$ \\
\hline \hline
\end{tabular}

$$
\xi_{\mu \mu^{\prime}}(s, t)=\left(\frac{1-z_{s}}{2}\right)^{\left|\mu-\mu^{\prime}\right| / 2}\left(\frac{1+z_{s}}{2}\right)^{\left|\mu+\mu^{\prime}\right| / 2},
$$

where $\mu=\mu_{1}-\mu_{2}$ and $\mu^{\prime}=\mu_{3}-\mu_{4}$ are the net helicity in the initial and final states, respectively. The half-angle factor incorporates the kinematic singularities in $t$ of the $s$-channel helicity amplitude, and in the forward direction at high energies it reduces to

$$
\xi_{\mu \mu^{\prime}}(s, t) \stackrel{s \rightarrow \infty}{\rightarrow} \sqrt{\frac{-t^{\mu-\mu^{\prime} \mid}}{s}} .
$$

By comparing with the asymptotic limit of the Regge pole expression given by Eq. (6), we obtain

$$
\begin{aligned}
A_{\mu_{4} \mu_{3} \mu_{2} \mu_{1}}= & \frac{\xi_{\mu \mu^{\prime}}(s, t)}{\sqrt{\frac{-t}{s}}\left|\mu-\mu^{\prime}\right|} \\
& \times\left[\sqrt{-t}\left|\mu_{1}-\mu_{3}\right| \sqrt{-t}\left|\mu_{2}-\mu_{4}\right| \hat{\beta}_{\mu_{1} \mu_{3}}^{e 13}(t) \hat{\beta}_{\mu_{2} \mu_{4}}^{e 24}(t) \mathcal{F}_{e}(s, t)\right],
\end{aligned}
$$

where the half-angle factor is fully taken into account. Alternatively, we can restore the half angle factor by multiplying the Regge formula Eq. (6) by a factor $\mathcal{R}$

$$
\mathcal{R}(s, t) \equiv\left(\frac{1-z_{s}}{2} \frac{\nu}{-t}\right)^{\frac{1}{2}\left|\mu-\mu^{\prime}\right|}\left(\frac{1+z_{s}}{2}\right)^{\frac{1}{2}\left|\mu+\mu^{\prime}\right|},
$$

where $\nu=(s-u) / 2$. It is worth noting that the small $|t|$ behavior imposed by angular-momentum conservation $\beta_{\mu_{4} \mu_{3} \mu_{2} \mu_{1}}^{e}(t) \propto \sqrt{-t}\left|\left(\mu_{1}-\mu_{3}\right)-\left(\mu_{2}-\mu_{4}\right)\right|$ in Eq. (8) is weaker than the behavior $\beta_{\mu_{4} \mu_{3} \mu_{2} \mu_{1}}^{e}(t) \propto \sqrt{-t}\left|\mu_{1}-\mu_{3}\right|+\left|\mu_{2}-\mu_{4}\right|$ introduced by the additional requirement of factorization of the residue in Eqs. (5) and (6). We have normalized $\mathcal{R}$ in such a way that $\mathcal{R} \rightarrow 1$ for $s \rightarrow \infty$, such that the $s$-dependence of the helicity amplitude remains $s^{\alpha_{e}(t)}$, as in Eq. (6). The final functional form for the amplitudes used for the fits reads

$$
\begin{aligned}
A_{\mu_{4} \mu_{3} \mu_{2} \mu_{1}}(s, t)= & \mathcal{R}(s, t) \sqrt{-t}^{\left|\mu_{1}-\mu_{3}\right|} \sqrt{-t}\left|\mu_{2}-\mu_{4}\right| \\
& \times \hat{\beta}_{\mu_{1} \mu_{3}}^{e 13}(t) \hat{\beta}_{\mu_{2} \mu_{4}}^{e 24}(t) \mathcal{F}_{e}(s, t) .
\end{aligned}
$$

Regge theory does not predict the full $t$-dependence, and the single-particle model only approximates the $t$-dependence close to the single particle pole. An exponential factor is introduced with a slope parameter, and in the following, we will implicitly assume the presence of an exponential factor in the residues

$$
\hat{\beta}_{\mu_{i} \mu_{j}}^{e i j}(t) \propto e^{b_{\mu_{i} \mu_{j}}^{e i j} t}
$$

The function $\mathcal{F}_{e}(s, t)$ is often referred to as the Regge propagator and is given by 
$\mathcal{F}_{e}(s, t)=-\frac{\zeta_{e} \pi \alpha_{e}^{1}}{\Gamma\left(\alpha_{e}(t)-l_{e}+1\right)} \frac{1+\zeta_{e} e^{-i \pi \alpha_{e}(t)}}{2 \sin \pi \alpha_{e}(t)}\left(\frac{s}{s_{0}}\right)^{\alpha_{e}(t)}$.

In the $t \rightarrow m_{e}^{2}$ limit, where $m_{e}$ is the mass of the meson with lowest spin $J_{e}$ on the trajectory $\alpha_{e}(t)$, one recovers the known single-particle propagator ${ }^{3}$

$$
\mathcal{F}_{e}(s, t) \underset{t \rightarrow m_{e}^{2}}{\rightarrow} \frac{\left(s / s_{0}\right)^{J_{e}}}{m_{e}^{2}-t} .
$$

This property illustrates that the Regge amplitude in Eq. (11) reduces to the single-particle exchange amplitude near the particle poles. This property constrains the residues near the pole to be numerically close to the phenomenological values from single-particle exchange models [34].

Note that in our approach the reggeons are used to directly construct the $s$-channel helicity amplitudes. A more natural approach would be to start in the rest frame of the reggeon, i.e., in the $t$-channel center-of-mass (c.m.) frame and apply crossing relations. In the $t$-channel frame, the relation between the residue zeros and angular momentum conservation is more transparent. Consider, for example, the $t$-channel amplitude $\pi \pi \rightarrow \bar{N} N$ with a $\rho$ exchange. A spin- 0 exchange is unphysical in the $t$-channel helicity flip component. Such a contribution is referred to as "nonsense" [9]. Therefore, a nonsense wrong-signature zero (NWSZ) is often introduced at $\alpha_{\rho}=0$ only in the $t$ channel helicity-flip component, in order to remove such a spurious contribution. For the $t$-channel nonflip component, the finite-energy sum rule analysis indeed shows a finite residue at the wrong signature point $\left(\alpha_{\rho}=0\right)$ [10]. However, starting from the $t$-channel, a global analysis is tedious and less straightforward, as discussed, for example, in [35]. Indeed, for each channel one must trace the kinematic singularities in $t$ and remove them in order to construct a set of helicity amplitudes which contain only dynamic singularities in $t$. In contrast, the $t$-singularities of the $s$-channel helicity amplitudes are easy to find as they originate entirely from the half-angle factors defined in Eq. (7); see Appendix A. By merely dividing out the halfangle factors, one is able to write down $s$-channel helicity amplitudes that are free from kinematic singularities in $t$. For a global phenomenological analysis, it is therefore preferential to deal directly with the $s$-channel amplitudes.

In this work, we will focus on the total and differential cross sections only. For most reactions, the amount of these

\footnotetext{
${ }^{3}$ Note that our convention is different from the one in [37] in the $\zeta_{e}$ prefactor. Our convention is correctly normalized to the single-particle pole as in Eq. (14), while the definition of [37] flips the sign according to the signature of the exchange. Naturally, this normalization affects the sign of the residues of the negative signature exchanges $\left(\zeta_{e}=-1\right)$. We absorb the sign difference into the top vertices of those exchanges.
}

type of data outweighs the polarization data count. Additionally, polarization observables are often measured at energies that are not high enough for our analysis. We restrict our model to the leading Regge pole contributions only. Polarization observables at high energies often function as a filter of specific $t$-channel quantum numbers and are sensitive to subtle interference effects in the amplitudes. Their interpretation is a study on its own. Since our goal is to obtain a qualitative description, we do not include such polarization data.

\section{CONSTRAINED SU(3)-EXD FIT}

The total number of parameters describing Regge residues is substantial. In order to obtain a robust estimate, we first carry out a fit to the data in which we impose both $\mathrm{SU}(3)$ and exchange degeneracy (EXD). Furthermore, we fix the $t$-dependence of the reduced residues $\hat{\beta}_{\mu_{i} \mu_{j}}^{e i j}$ (see Eq. (11) using a single-particle exchange model obtained from the effective Lagrangians as discussed in Appendices $\mathrm{C}$ and $\mathrm{E}$. We refer to this analysis as the "global SU(3)EXD fit", which is similar to the analyses of $[33,34]$. The results are shown in Sec. III B. We later relax all these assumptions for a second unconstrained fit in Sec. IV.

\section{A. Constraints}

To derive the SU(3) couplings, we consider SU(3) relations based on the Lagrangians in Appendix $\mathrm{C}$ for the single-particle exchange model. Using the relation between the single-particle and the Regge residues one obtains the SU(3) constraints for the latter, using the residues of the $\rho$ and $K^{*}$ exchanges ${ }^{4}$ as input. Since we do not consider reactions dominated by $\mathbb{P}$ or $\pi$ exchange, we do not fit $N N$ cross section data. If we did, it would allow us to constrain the overall normalization of the fit, through the determination of $\hat{\beta}^{e N N}$. Instead, we extract the top vertices from resonance decay widths. We fix $\hat{\beta}^{\rho \pi^{-} \pi^{-}}=$ 8.4 using the Lagrangian coupling, $g_{\mathrm{VPP}}=-4.2$, as discussed in Appendix D. Note that the sign is chosen such that the contributions to the total cross sections match correctly. While SU(3) allows one to relate the various Regge residues of vector exchanges, it does not relate these to residues of tensor exchanges $\left(a_{2}\right.$ and $\left.K_{2}^{*}\right)$. The $a_{2}$ and $K_{2}^{*}$ couplings for the global SU(3)-EXD fit are obtained by demanding EXD for the helicity residues. In summary, we use the following relations obtained from duality arguments [6]

$$
\begin{gathered}
\hat{\beta}^{\rho K^{+} K^{+}}=-\hat{\beta}^{a_{2} K^{+} K^{+}}, \\
\hat{\beta}^{\rho p p}=\hat{\beta}^{a_{2} p p},
\end{gathered}
$$

\footnotetext{
${ }^{4}$ We denote reggeons by their lowest spin particles. Also, $K^{*} \equiv$ $K^{*}(892)$ and $K_{2}^{*} \equiv K_{2}^{*}(1430)$.
} 


$$
\hat{\beta}^{\rho p \Delta^{+}}=\hat{\beta}^{a_{2} p \Delta^{+}}
$$

for any helicity combination. Since $K^{+} p \rightarrow K^{+} p$ and $K^{+} n \rightarrow K^{+} n$ are exotic in the $s$-channel, duality requires that $\hat{\beta}^{\rho K^{+} K^{+}} \hat{\beta}^{\rho p p}=-\hat{\beta}^{a_{2} K^{+} K^{+}} \hat{\beta}^{a_{2} p p}$ since the propagator is normalized according to Eq. (14) (and similarly for $f$ and $\omega$ exchanges). Note that exact EXD is not necessarily fulfilled within the single-particle model. In particular, EXD requires the residues of two exchanges to be equal for any $t$, which might not be possible, especially when only a subset of the interaction Lagrangians is considered, as is often the case in the literature. Therefore, an "EXD propagator" (which amounts to adding or subtracting the propagators of EXD contributions) is usually introduced to circumvent this issue, while strong EXD is in fact a property of the residues. In the latter approach, one effectively assumes that both exchanges have the singleparticle residue of the lowest spin exchange.

It is worth noting that EXD is sometimes used incorrectly. It is interpreted as a property of a single reggeon, while it is in fact a relation between different Regge pole contributions. Therefore, the EXD propagator can only be used in reactions where both EXD partners are allowed. For instance, there is no strong EXD for the residues $\beta_{\mu_{\gamma} \mu_{\pi}}^{e \gamma \pi}(t)$ of $\pi$ photoproduction reactions since there is no exotic $s$-channel reaction containing these residues.

In the global SU(3)-EXD fit, we opt to introduce a single exponential damping factor for each independent helicity configuration $b_{\mathrm{nf}}, b_{\mathrm{sf}}$, with $\mathrm{nf}$ and sf referring to helicity nonflip and single-flip amplitudes. These are fitted to the data. In this fit, we set the double-flip amplitudes to zero.

\section{B. Results}

In the following, we present results of the global analysis of the high-momentum $\left(p_{\text {lab }} \geq 5 \mathrm{GeV}\right)$ differential cross section data for a large number of reactions, including reactions with $\pi$ and $K$ beams that are dominated by $\rho, a_{2}$, $K^{*}$, and $K_{2}^{*}$ exchanges. ${ }^{5}$ Hereby, we consider the channels with strangeness and charge exchange (CEX). The data used in this analysis are listed in Table II.

The $\Gamma$ function in Eq. (4) introduces zeros in the cross sections for reactions dominated by the $\rho$ exchange, e.g., $\pi^{-} p \rightarrow \pi^{0} n$. In this case, daughter poles or other subleading singularities become relevant as they tend to fill in the zeros $[34,76]$. In the SU(3)-EXD, we focus only on the leading natural Regge poles and do not include any subleading Regge contribution. Instead of introducing new amplitude components to fill in the dips, we prefer to work with the well-defined Regge pole model. Therefore, we must reduce the contribution from dip regions in $\rho$-only dominated channels in Figs. 4 and $8 \mathrm{a}$,

\footnotetext{
${ }^{5} \mathrm{We}$ do not report fits for tensor meson production since the scarceness of the data strongly hinders a reliable fit.
}

TABLE II. Available differential cross section data and references.

\begin{tabular}{lc}
\hline \hline Reaction & Data references \\
\hline$\pi^{+} p \rightarrow X$ & {$[38]$} \\
$K^{ \pm} N \rightarrow X$ & {$[38]$} \\
$\pi^{-} p \rightarrow \pi^{0} n$ & {$[39,40]$} \\
$\pi^{+} p \rightarrow \pi^{0} \Delta^{++}$ & {$[41-44]$} \\
$\pi^{-} p \rightarrow \eta n$ & {$[45-48]$} \\
$\pi^{-} p \rightarrow \eta^{\prime} n$ & {$[48,49]$} \\
$\pi^{+} p \rightarrow \eta \Delta^{++}$ & {$[41,42]$} \\
$K^{+} n \rightarrow K^{0} p$ & {$[50-52]$} \\
$K^{-} p \rightarrow \bar{K}^{0} n$ & {$[50-59]$} \\
$K^{+} p \rightarrow K^{0} \Delta^{++}$ & {$[51,52,57,60,61]$} \\
$K^{-} n \rightarrow \bar{K}^{0} \Delta^{-}$ & {$[51,52]$} \\
$K^{-} p \rightarrow \bar{K}^{0} \Delta^{0}$ & {$[57,59]$} \\
$K^{-} p \rightarrow \pi^{-} \Sigma^{*+}$ & {$[58,62-64]$} \\
$K^{-} p \rightarrow \pi^{-} \Sigma^{+}$ & {$[58,62,63,65]$} \\
$K^{-} p \rightarrow \pi^{0} \Lambda$ & {$[58,66]$} \\
$\pi^{+} p \rightarrow K^{+} \Sigma^{*+}$ & {$[62,63,67]$} \\
$\pi^{+} p \rightarrow K^{+} \Sigma^{+}$ & {$[62,63,65,67,68]$} \\
$\pi^{-} p \rightarrow K^{0} \Lambda$ & {$[69-71]$} \\
$\pi^{-} p \rightarrow K^{0} \Sigma^{0}$ & {$[69-71]$} \\
$K^{-} p \rightarrow \eta \Lambda$ & {$[58,66]$} \\
$K^{-} p \rightarrow \eta^{\prime} \Lambda$ & {$[58,66]$} \\
$\pi^{-} p \rightarrow \omega n$ & {$[72-74]$} \\
$\pi^{+} n \rightarrow \omega p$ & {$[75]$} \\
\hline \hline
\end{tabular}

by rescaling the data error bars using a factor $f(t)=$ $1 /\left|t-t_{0}\right|$, where $t_{0}$ is defined such that $\alpha_{\rho}\left(t_{0}\right)=0$. Such a rescaling is required in order for the fit to be less sensitive to kinematic regions dominated by components beyond the leading pole model. The goal of this work is to detect the regions where the Regge pole approximation holds best by manually varying the kinematic regions. Additionally, the available data are subject to sizable and uncontrolled systematic errors. Therefore, we aim to provide a qualitative description and do not report the $\chi^{2}$ values and errors of the fits.

The global SU(3)-EXD fit contains nine free parameters, which are given in Table III. The other three are the $\eta-\eta^{\prime}$

TABLE III. Reduced SU(3) couplings obtained from a global SU(3)-EXD fit. Fixed couplings are indicated by an asterisk.

\begin{tabular}{lcc}
\hline \hline Top vertices & Bottom vertices & Damping $\left(\mathrm{GeV}^{-2}\right)$ \\
\hline$\theta_{P}=-0.14$ & $g_{\mathrm{VBB}}^{v, D}=-1.29$ & $b_{\mathrm{sf}}=0.54$ \\
$g_{\mathrm{VPP}}=-4.2^{*}$ & $g_{\mathrm{VBB}}^{v, F}=2.35$ & $b_{\mathrm{nf}}=1.31$ \\
$g_{V V P}=45.25 \mathrm{GeV}^{-2}$ & $g_{\mathrm{VBB}}^{t, D}=6.93$ & \\
& $g_{\mathrm{VBB}}^{t, F}=3.64$ & \\
& $g_{\mathrm{VBD}}^{(1)}=-7.11$ & \\
\hline
\end{tabular}




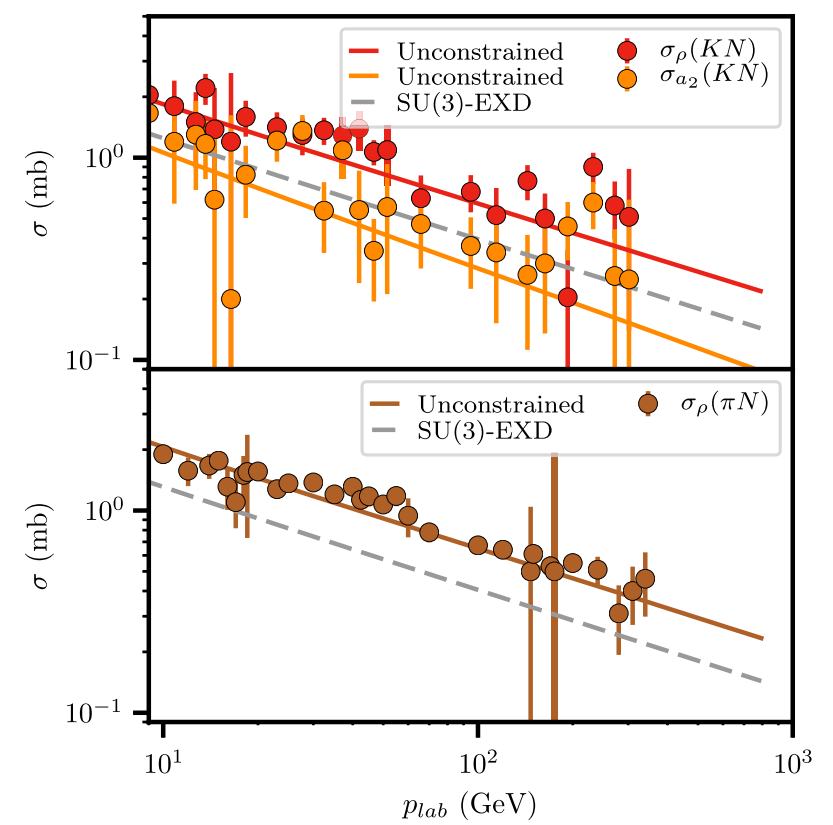

FIG. 3. Total cross section combinations in Eqs. (18a)-(18c). The dashed and solid lines represent the global SU(3)-EXD and isospin constrained fit, respectively.

mixing angle $\theta_{P}$ and the single- and nonhelicity-flip exponential factors $b_{s f}$ and $b_{n f}$, respectively. In total, $N_{\text {data }}=1271$ high-energy forward scattering data points are fitted, with $p_{\text {lab }} \geq 5 \mathrm{GeV}$ and $0 \leq-t \leq 0.8 \mathrm{GeV}^{2}$. For the total cross sections, we consider data at slightly higher energies, $p_{\text {lab }} \geq 10 \mathrm{GeV}$, since the Regge-based model in [38] matches best above this energy. The parameters of the natural Regge trajectories are fixed to the values given in Table I. Since there are too many amplitudes describing the production of spin $3 / 2$ baryons compared to the available data, additional constraints are needed. Specifically, we keep only a single term in the interaction Lagrangian of vector-meson-octet-baryon-decuplet-baryon couplings (VBD), i.e., we set $g_{\mathrm{VBD}}^{(2)}=g_{\mathrm{VBD}}^{(3)}=0$ in Eq. (C19) for all VBD combinations. More details are given in Appendix C. Note that this sets all double flip components to zero, in agreement with the data. Additionally, it is worth mentioning that the reduced residue of the nonflip component is proportional to $t$ in the single-meson form in Table VII. Even though this is not required by factorization and angular-momentum conservation, the contribution is therefore required to vanish at $t=0$ in the single-meson exchange approximation.

The comparison between the data and the model is shown in Figs. 3-13. The global SU(3)-EXD fit is stable and provides a remarkably good description of all the key features in the data. In Table IV we list all exchanges that contribute to the reactions we have analyzed and values of the residues derived from the fit. We do not take into account the exponential factor in Eq. (12) when we extrapolate the residues to the pole, as it is expected to be a fair approximation in the physical region only. This will allow us to directly relate our extracted couplings to those in modern literature as discussed in Appendix D. The residues are computed from the couplings in Table III.

In the following, we discuss in detail the model predictions for the various channels. We consider first the appropriate combinations of total cross sections which are

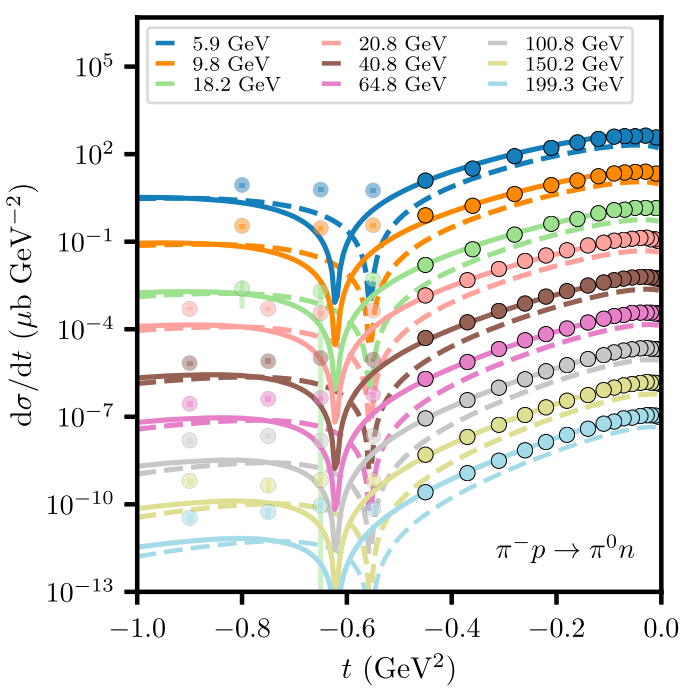

(a) $\pi^{-} p \rightarrow \pi^{0} n$

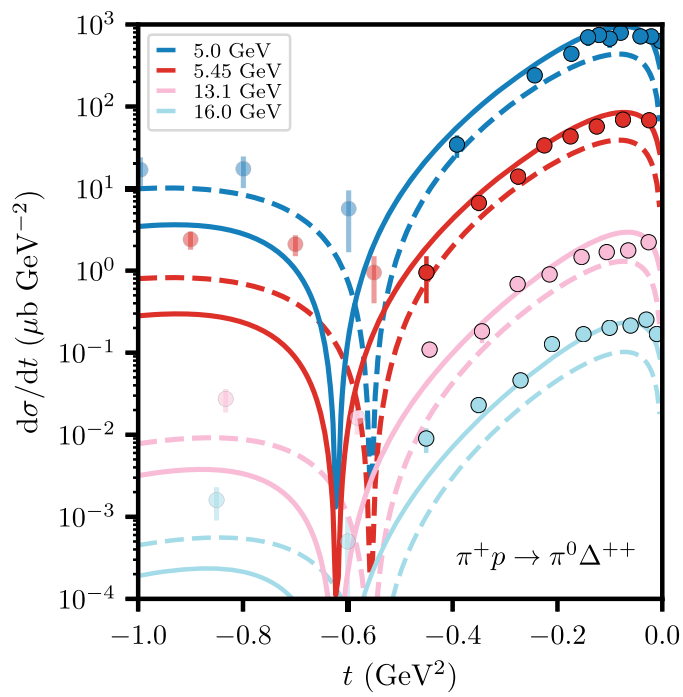

(b) $\pi^{+} p \rightarrow \pi^{0} \Delta^{++}$

FIG. 4. Differential cross section for the channels dominated by $\rho$ exchange. Dashed (solid) lines represent the global SU(3)-EXD (unconstrained) fit. The cross section for the lowest $p_{\text {lab }}$ has not been rescaled. For momentum value $i$ (in ascending order, with $i=0$ the lowest $p_{\text {lab }}$ ), the cross section has been rescaled by a factor $(0.1)^{i}$. The legend shows the $p_{\text {lab }}$ values of the measurements. The transparent data set has not been included in the unconstrained fit but are shown for completeness. 


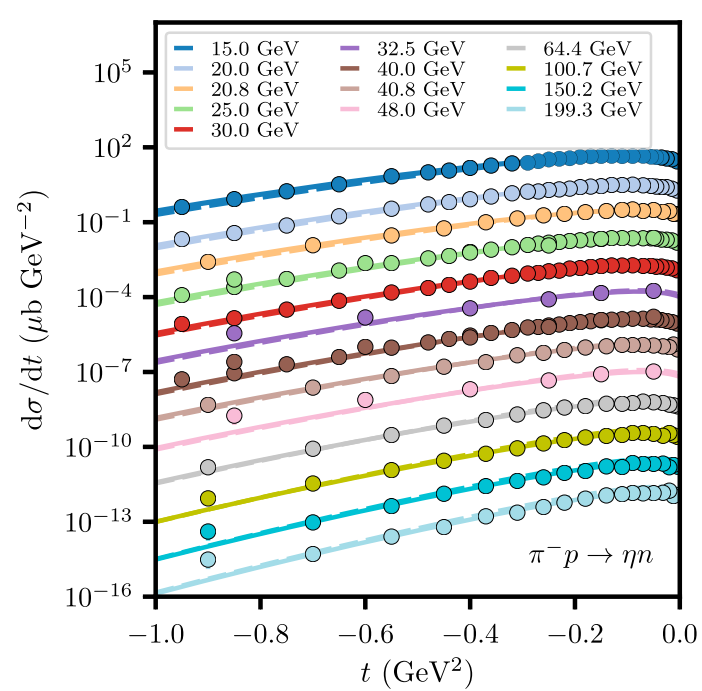

(a) $\pi^{-} p \rightarrow \eta n$

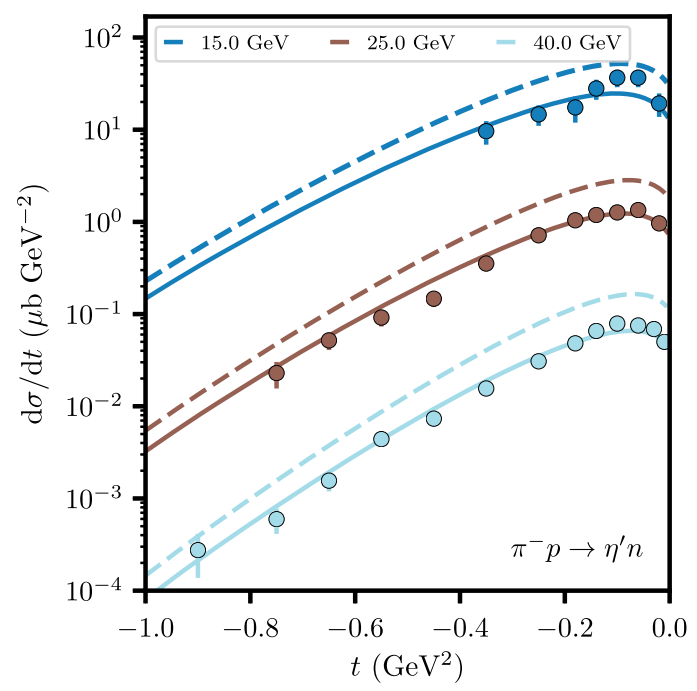

(b) $\pi^{-} p \rightarrow \eta^{\prime} n$

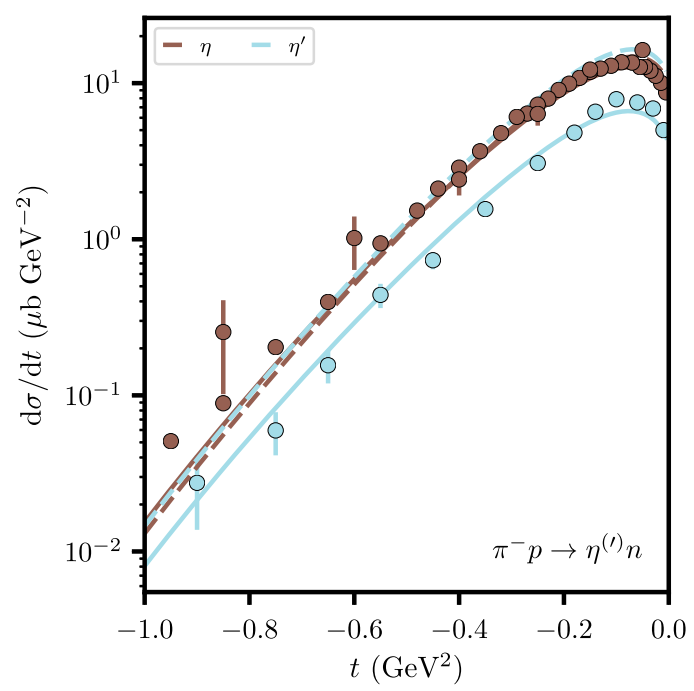

(c) $\pi^{-} p \rightarrow \eta^{(\prime)} n$

FIG. 5. Differential cross section for the channels dominated by $a_{2}$ exchange only. Scaling and conventions are as in Fig. 4. Figure 5c shows $\eta^{(\prime)} n$ for $p_{\text {lab }}=40 \mathrm{GeV}$.

sensitive to $\rho$ and $a_{2}$ exchanges. The optical theorem relates the total cross section to the elastic amplitude at $t=0$ via

$$
\sigma(1+2 \rightarrow X)=\frac{\sum_{\mu_{1} \mu_{2}} \operatorname{Im} A_{\mu_{2} \mu_{1} \mu_{2} \mu_{1}}(s, t=0)}{\left(2 s_{1}+1\right)\left(2 s_{2}+1\right) S_{12}(s)}
$$

where $S_{i j}$ is a kinematic function defined in Appendix A. The contribution from the individual Regge poles to the elastic amplitudes in Eq. (16) is given by

$$
\begin{gathered}
A\left(\pi^{ \pm} p\right)=\mathbb{P}+f_{2} \mp \rho, \\
A\left(K^{ \pm} p\right)=\mathbb{P}+f_{2} \mp \rho+a_{2} \pm \omega,
\end{gathered}
$$

$$
A\left(K^{ \pm} n\right)=\mathbb{P}+f_{2} \pm \rho-a_{2} \pm \omega .
$$

Hence, defining the following linear combinations,

$$
\begin{aligned}
\sigma_{\rho}(K N)= & \sigma\left(K^{-} p\right)-\sigma\left(K^{-} n\right) \\
& -\sigma\left(K^{+} p\right)+\sigma\left(K^{+} n\right), \\
\sigma_{a_{2}}(K N)= & \sigma\left(K^{-} p\right)-\sigma\left(K^{-} n\right) \\
& +\sigma\left(K^{+} p\right)-\sigma\left(K^{+} n\right), \\
\sigma_{\rho}(\pi N)= & \sigma\left(\pi^{-} p\right)-\sigma\left(\pi^{+} p\right),
\end{aligned}
$$

one can investigate the individual contributions from the $\rho$ and $a_{2}$ exchanges. In the case of exact SU(3) and EXD, all three cross section combinations must be equal. Inspecting 


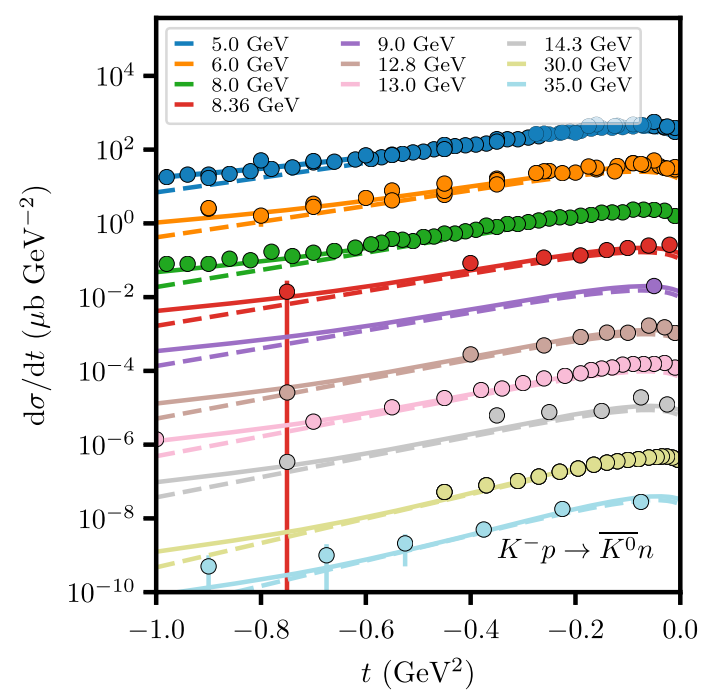

(a) $K^{-} p \rightarrow \overline{K^{0}} n$

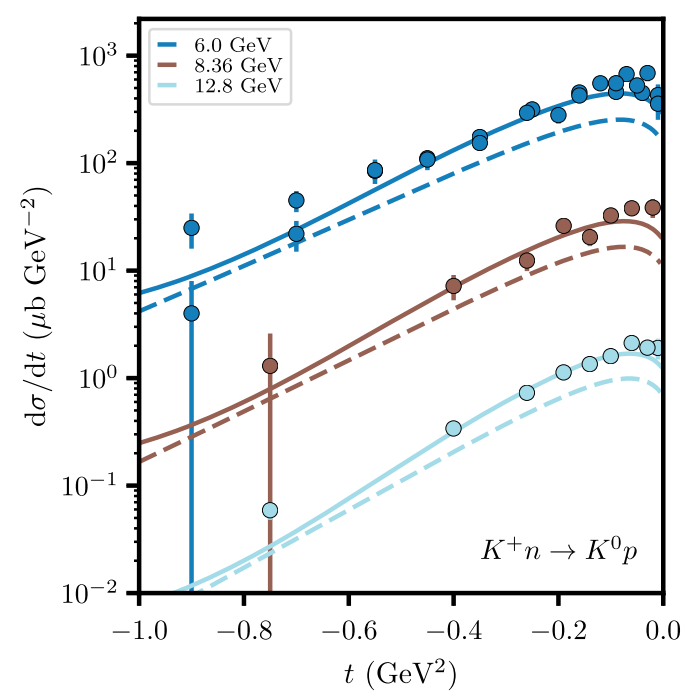

(b) $K^{+} n \rightarrow K^{0} p$

FIG. 6. $K N \rightarrow K N$ CEX reaction data. Scaling and conventions are as in Fig. 4.

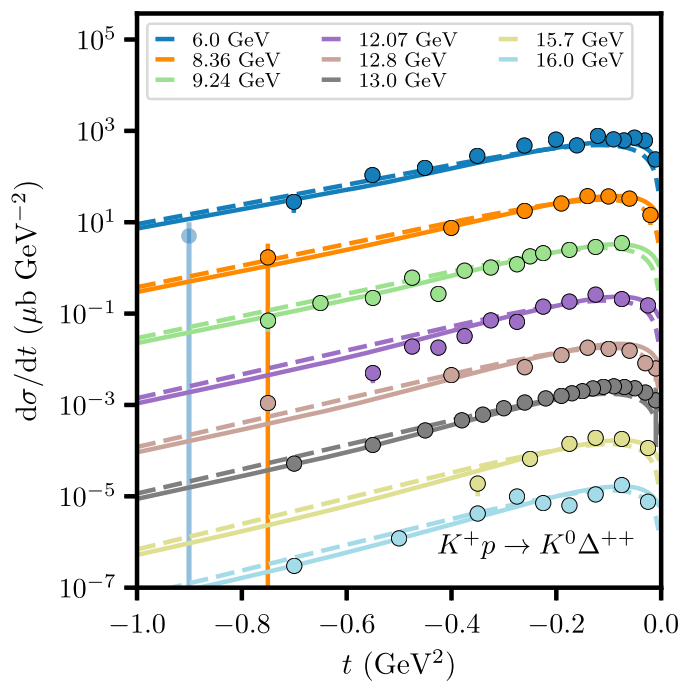

(a) $K^{+} p \rightarrow K^{0} \Delta^{++}$

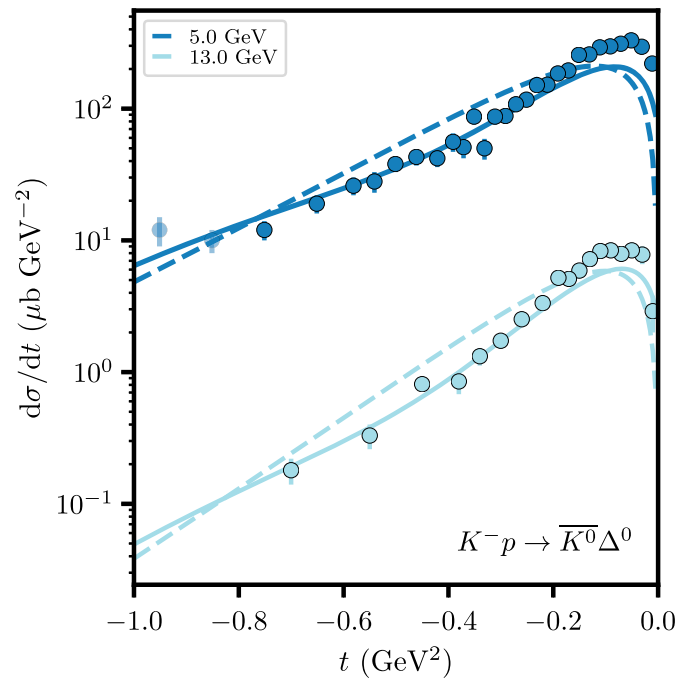

(b) $K^{-} p \rightarrow \overline{K^{0}} \Delta^{0}$

FIG. 7. $K N \rightarrow K \Delta$ CEX reaction data. Scaling and conventions are as in Fig. 4.

the results shown in Fig. 3, one sees that $\sigma_{\rho}(K N)$ and $\sigma_{a_{2}}(K N)$ differ by a few mb only, indicating a small violation of exchange degeneracy. The compatibility of $\sigma_{\rho}(K N)$ and $\sigma_{\rho}(\pi N)$ illustrates that the $\mathrm{SU}(3)$ symmetry is well respected in $K N$ and $\pi N$ scattering.

The $\Gamma$ function in Eq. (13) is introduced to remove spurious spin exchange components. The spin 0 exchange term in the $a_{2}$ trajectory at $\alpha_{a_{2}}(t)=0$ corresponds to an unphysical pole with negative mass squared. As discussed earlier, due to EXD, a zero in the residue for $a_{2}$ exchange forces a zero in the residue of the $\rho$ exchange, even though the Regge propagator for the $\rho$ does not contain a pole at $\alpha_{\rho}(t)=0$. The residue zero results in the vanishing of the cross section, which can be observed in all reactions where $\rho$ exchange dominates, i.e., in $\pi N \rightarrow \pi N$ and $\pi N \rightarrow \pi \Delta$ in Fig. 4.

The SU(3) predictions for $\eta^{(\prime)}$ production are depicted in Figs. 5a and 5b, and an overlay of both reactions for $p_{\text {lab }}=40 \mathrm{GeV}$ is given in Fig. 5c. The comparison between $\eta$ and $\eta^{\prime}$ fits can be used to extract information on the pseudoscalar mixing. Using SU(3) constraints, one estimates the relative couplings as (see Appendix C)

$$
\frac{g_{a_{2} \pi \eta^{\prime}}}{g_{a_{2} \pi \eta}}=\frac{S_{T} \cos \theta_{P}+\sin \theta_{P}}{\cos \theta_{P}-S_{T} \sin \theta_{P}} .
$$

As discussed in Appendix C, the Okubo-Zweig-Iizuka (OZI) $s \bar{s}$ suppression rule requires that the relative coupling 


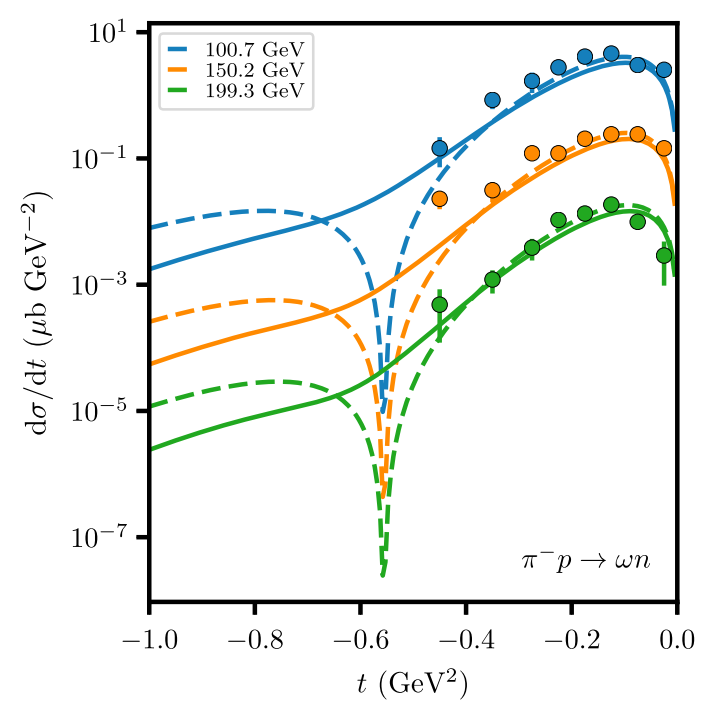

(a) $\pi^{-} p \rightarrow \omega n, p_{\text {lab }}>100 \mathrm{GeV}$

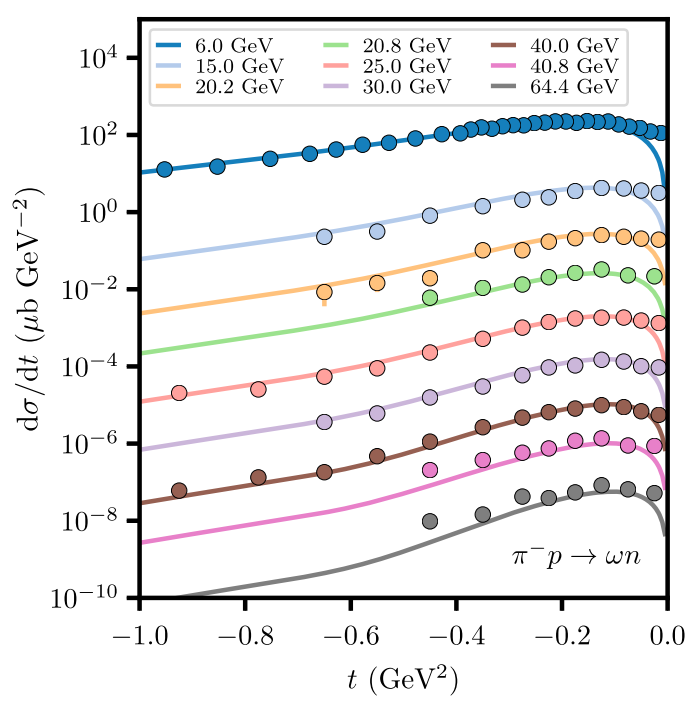

(b) $\pi^{-} p \rightarrow \omega n, p_{\text {lab }}<100 \mathrm{GeV}$

FIG. 8. Results for the CEX $\omega$ production channels. Scaling and conventions are as in Fig. 4.

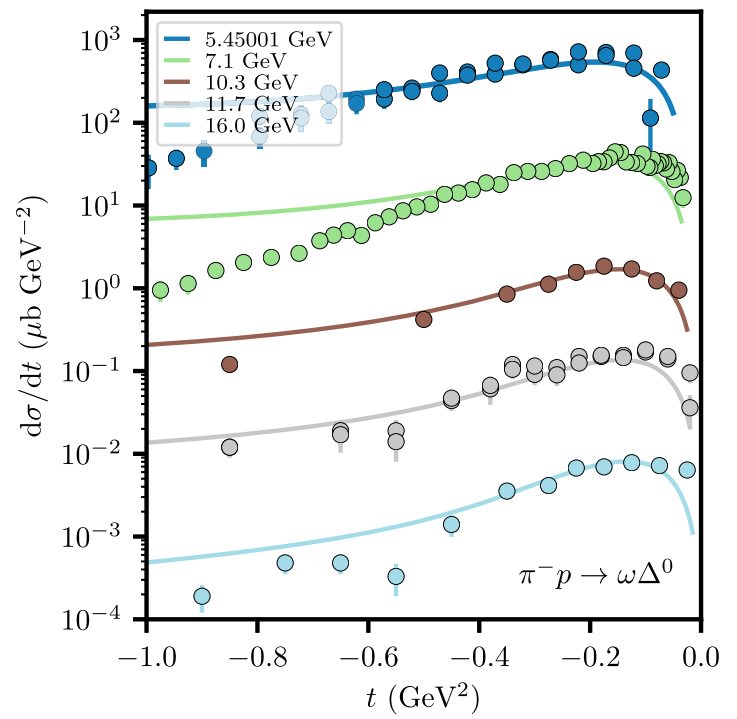

FIG. 9. Results for the CEX $\omega$ production channel $\pi^{-} p \rightarrow \omega \Delta^{0}$. Scaling and conventions are as in Fig. 4.

of the singlet $\eta_{1}$ and octet $\eta_{8}$ components is given by $S_{T}=\sqrt{2}$. Hence, for mixing angles $\theta_{P} \approx-0.17$ and under the OZI assumption $\left(S_{T}=\sqrt{2}\right)$, one finds $g_{a_{2} \pi \eta^{\prime}} \approx g_{a_{2} \pi \eta}$. The exact size of the pseudoscalar mixing angle $\theta_{P}$ is unknown, but the various theoretical estimates suggest values in the range $-0.38 \lesssim \theta_{P} \lesssim-0.17$ [77-82].

The fit results in a good correspondence to $K N \rightarrow K N$ and $K N \rightarrow K \Delta$ CEX in Figs. 6 and 7. In these reactions, both $\rho$ and $a_{2}$ contribute. While the $\rho$ dominates the very forward region, the $a_{2}$ exchange fills up the dip of the $\rho$ in the neighborhood of $\alpha_{\rho}(t)=0$.

For $\omega N$ production at very high energies in Fig. 8a, a fit with only $\rho$ exchange shows the correct $s$-dependence over a wide energy range. The data also clearly show a dipping behavior near $\alpha_{\rho}(t)=0$, as expected for a pure $\rho$ contribution. The data on $\omega$ production in Fig. 8 are dominated by $\rho$ exchange at very high energies $p_{\text {lab }} \geq 100 \mathrm{GeV}$ since in the forward direction, the $b_{1}$ exchange contribution is suppressed by a factor of $s^{-1 / 2}$ in the amplitudes, relative to the natural exchange. In the fits, we wish to determine the residues of only the leading Regge poles that are constrained by multiple channels. Therefore, we consider only $p_{\text {lab }} \geq 100 \mathrm{GeV}$ to isolate the $\rho$ component and neglect the $b_{1}$ exchange. The data on $\omega \Delta$ production are rather scarce and at energies sensitive to the $b_{1}$ exchange (see Fig. 9). Therefore, we do not consider them in the global SU(3)CEX fit.

Finally, we consider strangeness exchange in Figs. 10-13. The effective trajectories ${ }^{6}$ obtained in [70] from $\Lambda$ and $\Sigma^{0}$ production data are much flatter than the ones compatible with $\alpha_{K^{*}}\left(t=m_{K^{*}}^{2}\right)=1$ and $\alpha_{K^{*}}\left(t=m_{K_{2}^{*}}^{2}\right)=2$ used in this work. They obtain $\alpha_{\text {eff }}^{0}=0.32$ and $\alpha_{\text {eff }}^{1}=0.23-0.43 \mathrm{GeV}^{-2}$. This disagreement indicates that secondary contributions (such as additional poles) are present at higher $-t$. The global fit indeed does not reproduce the high- $|t|$ region. However, a very good agreement is found in the very forward region. This kinematic domain follows the $s$-dependence compatible with our trajectories in Table I.

${ }^{6}$ The effective trajectories are obtained by fitting $\alpha_{\text {eff }}(t)$

$$
\frac{\mathrm{d} \sigma}{\mathrm{d} t}=f(t)\left(\frac{s}{s_{0}}\right)^{2 \alpha_{\mathrm{eff}}(t)-2}
$$

to the $s$-dependence at fixed $t$, where $f(t)$ is a fitting parameter which may be different for all $t$. 


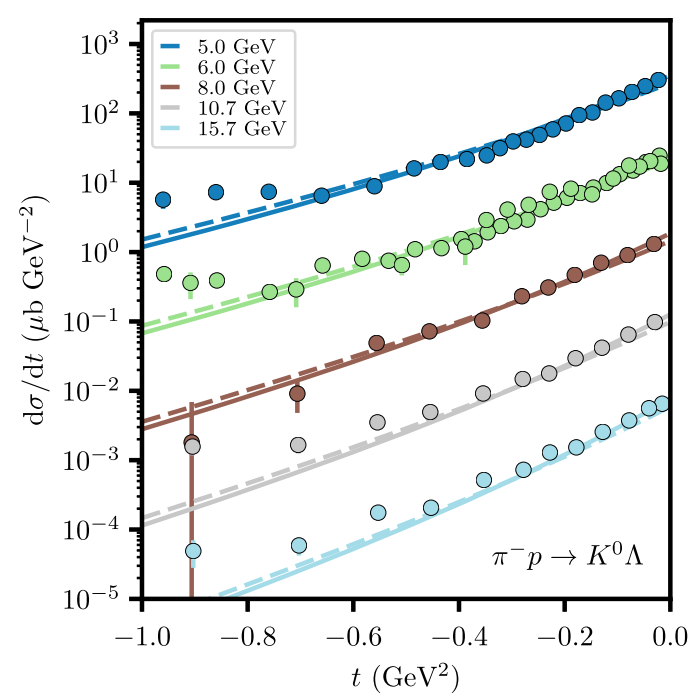

(a) $\pi^{-} p \rightarrow K^{0} \Lambda$

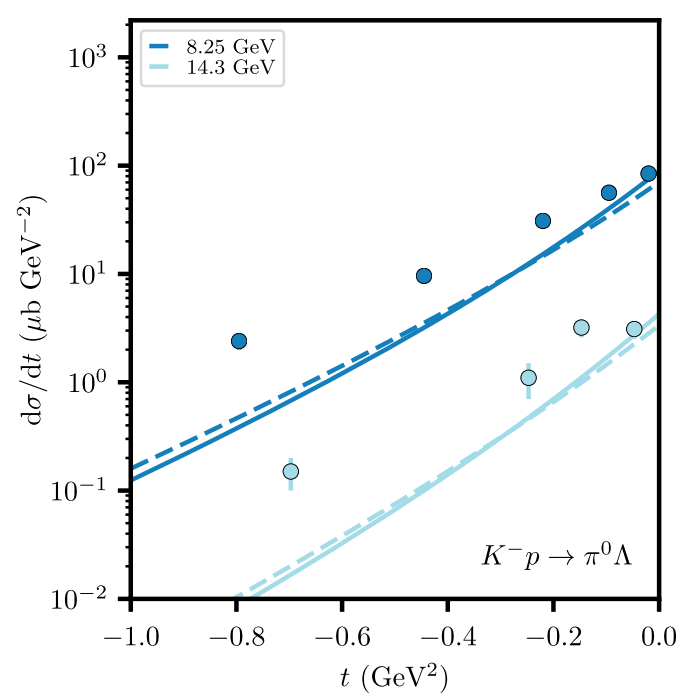

(b) $K^{-} p \rightarrow \pi^{0} \Lambda$

FIG. 10. Results for the strangeness exchange $\pi \Lambda$ and $K \Lambda$ production channels. Scaling and conventions are as in Fig. 4.

For both $\Lambda$ and $\Sigma$ production, an inconsistency is observed for the time-reversal related reactions in Figs. 10 and 11 respectively. The mismatch is related to the time-reversal symmetry of the $\beta^{K_{(2)}^{*} \pi K}(t)$ vertex imposed in the fit [34]. The latter requires $\beta^{K_{(2)}^{*} \pi K}(t)=-\beta^{K_{(2)}^{*} K \pi}(t)$, while the bottom vertex remains the same. Under the assumption of EXD, the cross section of the time-reversed reactions are therefore expected to be the same. The SU(3) relations force the $\Lambda$ (and to a lesser extent $\Sigma$ ) production to be dominated by helicity nonflip contributions in the forward direction. ${ }^{7}$ Note that the nonflip contributions respect the EXD and SU(3) relations very well.

\section{UNCONSTRAINED FIT}

In the unconstrained fit, we relax the EXD and SU(3) constraint and only keep SU(2), isospin, as a good symmetry. We also fit the Regge trajectory parameters of the $\rho$ and $a_{2}$ exchange. In this fit, we can keep the singleparticle approximation for the residues and fit the couplings in Table VII (without their SU(3) decomposition) for both vector and tensor exchanges. Indeed, when the EXD constraint is removed, the residues of the tensor exchanges must be determined independently. However, this approach is quite cumbersome. Additionally, this approach forces the residues to be restricted to the single-particle exchange residue. The tensor poles are quite far away from the physical region, and it can no longer be expected that this approximation is reliable in the physical region. Therefore, in our next fit the $t$-dependence of the residues is no longer

\footnotetext{
${ }^{7}$ Care must be taken when interpreting the pole couplings in Table IV: since the $t$-dependence of the different helicity couplings differs, the relative size of the residues in the physical region might be quite different from pole values.
}

constrained by the single-particle model, and we use Eq. (20) instead. Hereby, the reduced residues are parametrized as

$$
\hat{\beta}_{\mu_{i} \mu_{f}}^{e i f}(t)=g_{\mu_{i} \mu_{f}}^{e i f} e^{b_{\mu_{i} \mu_{f}}^{e i f} t}
$$

where the constants $g_{\mu_{i} \mu_{f}}^{e i f}$ and $b_{\mu_{i} \mu_{f}}^{\text {eif }}$ are all fitted independently, unless stated otherwise. The fitted residue parameters are tabulated in Tables V and VI.

Abandoning the strict connection with the particle exchange model also implies, for example, that the nonflip component of the VBD coupling is no longer required to vanish at $t=0\left(\right.$ see $\hat{\beta}_{+\frac{1}{2}+\frac{1}{2}}^{\mathrm{VBD}} \propto t$ for the single-particle residue in Table VII). All this significantly increases the number of parameters from 9 to 110 , and we fit them in steps including a few reactions at the time. The $t$-dependence in our fit is now entirely absorbed into the exponential factor.

Next, we describe the step-wise fitting process. We take advantage of the fact that a given exchange is related to a limited set of reactions. In the first step, we determine the trajectory intercepts, $\alpha_{\rho}^{0}, \alpha_{a_{2}}^{0}$, and the parameters of the residues, $\hat{\beta}_{++}^{\rho N N}, \hat{\beta}_{++}^{a_{2} N N}, \hat{\beta}_{-+}^{\rho N N}, \hat{\beta}_{-+}^{a_{2} N N}, \hat{\beta}^{\rho K K}, \hat{\beta}^{a_{2} K K}, \hat{\beta}^{\rho \pi \eta^{(\prime)}}$, and $\hat{\beta}^{a_{2} \pi \eta^{(\prime)}} c f$. Eq. (12). The slopes $\alpha_{\rho}^{1}\left(\alpha_{a_{2}}^{1}\right)$ follow from the requirement that $\alpha_{\rho}\left(t=m_{\rho}^{2}\right)=1\left(\alpha_{a_{2}}\left(t=m_{a_{2}}^{2}\right)=2\right)$. The intercepts are allowed to vary in the range $0.4 \leq \alpha_{\left(\rho, a_{2}\right)}^{0} \leq 0.55$. The results of these fits are depicted in Figs. 3, 4a, 5, and 6 Assuming $S_{T}=\sqrt{2}$, we obtain the mixing angle $\theta_{P}=-0.33$. This angle is compatible with the values found in the recent literature $[81,82]$. Inspecting the results in Fig. 5a one finds that the cross section rises rapidly for $-t \geq 0.8 \mathrm{GeV}^{2}$ and cannot be described within 


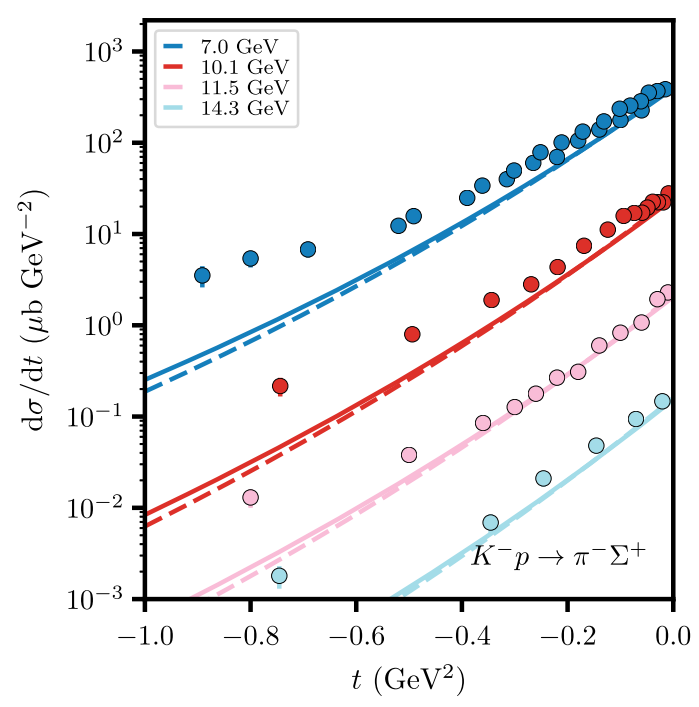

(a) $K^{-} p \rightarrow \pi^{-} \Sigma^{+}$

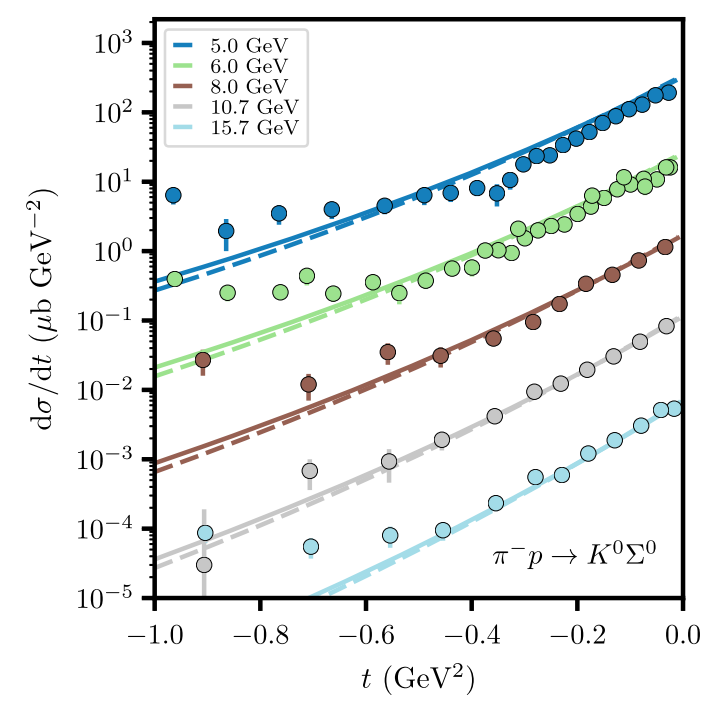

(b) $\pi^{-} p \rightarrow K^{0} \Sigma^{0}$

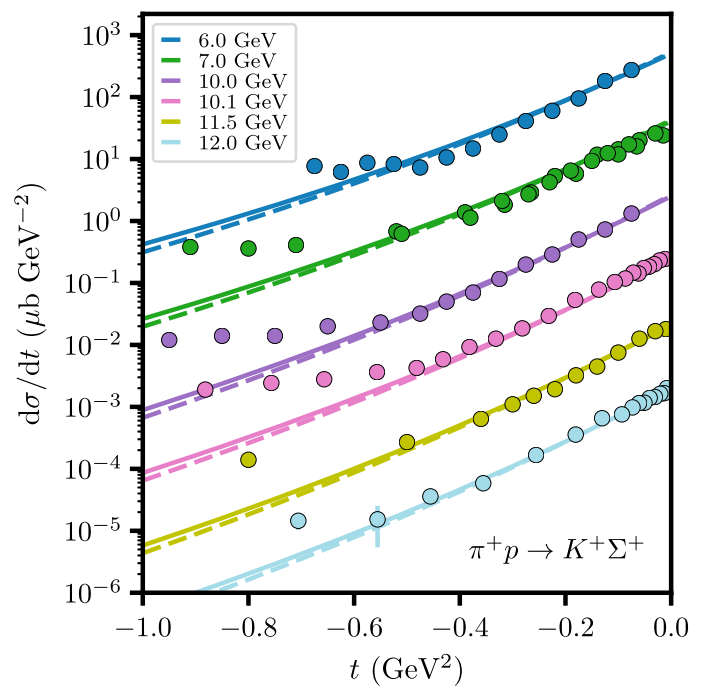

(c) $\pi^{+} p \rightarrow K^{+} \Sigma^{+}$

FIG. 11. Results for the strangeness exchange $\pi \Sigma$ and $K \Sigma$ production channels. Scaling and conventions are as in Fig. 4.

the pure Regge-pole picture. Therefore, this kinematic domain is excluded in the channels that follow.

With the couplings at the top vertex determined through the residues listed above, we proceed to determine the bottom couplings, i.e., $\hat{\beta}_{\mu_{N} \mu_{\Delta}}^{\rho N \Delta}$ and $\hat{\beta}_{\mu_{N} \mu_{\Delta}}^{a_{2} N \Delta}$, from a combined fit to $\pi N \rightarrow \eta \Delta, \pi N \rightarrow \pi \Delta$, and $K N \rightarrow K \Delta$ cross sections. The large number of helicity couplings leads to a rather unconstrained fit, and thus, based on the result of the SU(3)-EXD fit, we eliminate the double-flip components. Furthermore, we keep the ratio of the two single-flip components $g_{+\frac{1}{2}+\frac{3}{2}}^{e N \Delta} / g_{-\frac{1}{2}+\frac{1}{2}}^{e N \Delta}\left(e=\rho, a_{2}\right)$ fixed to the value obtained from the SU(3)-EXD fit. Their exponential $t$-dependence is assumed to be the same and is fitted to the data. The $t$-dependence of the nonflip components is fixed to the SU(3)-EXD values. The results of the fit are depicted in Figs. 4b, and 7. Relaxing the condition $\hat{\beta}_{+\frac{1}{2}+\frac{1}{2}}^{\rho N \Delta}(t=0)=0$ imposed by the single-particle exchange correspondence in Table VII seems to slightly improve the fit at forward angles. This effect is even clearer for $\Sigma^{*}$ production channels in Fig. 13.

Using the $\hat{\beta}^{\rho N N}$ couplings extracted in the previous fitting steps, one can now determine the $\hat{\beta}^{\rho \pi \omega}$ residue from a fit to the $\omega N$ production data at very high energies. At forward angles and low energies, the $b_{1}$ exchange does not represent the full strength required to reproduce the cross section of $\omega$ production. Notice that the NWSZ at $\alpha_{b_{1}}(t)=0$ would force the cross section to vanish near $t \approx 0$. This mismatch is typically associated with the existence of a trajectory with quantum numbers $I^{G \zeta \eta}=1^{++-}$, with the lowest spin meson located on the trajectory being the yet 


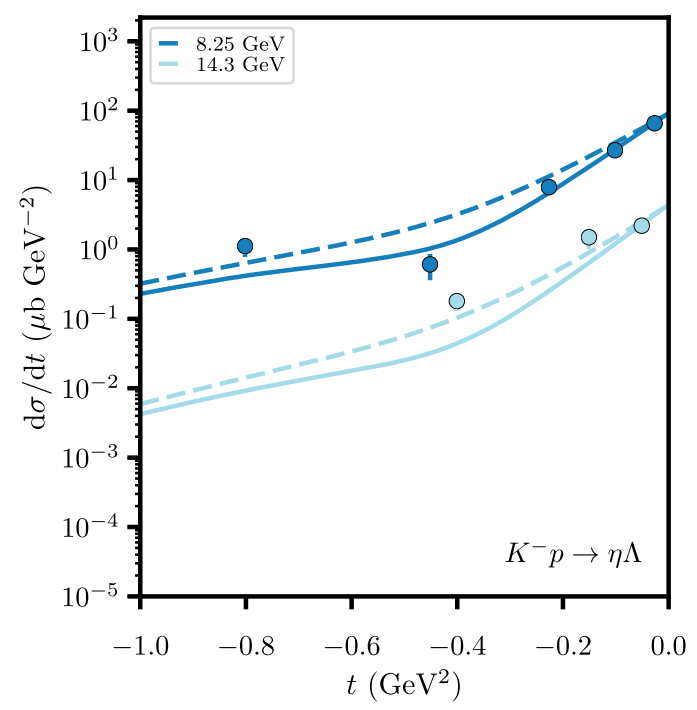

(a) $K^{-} p \rightarrow \eta \Lambda$

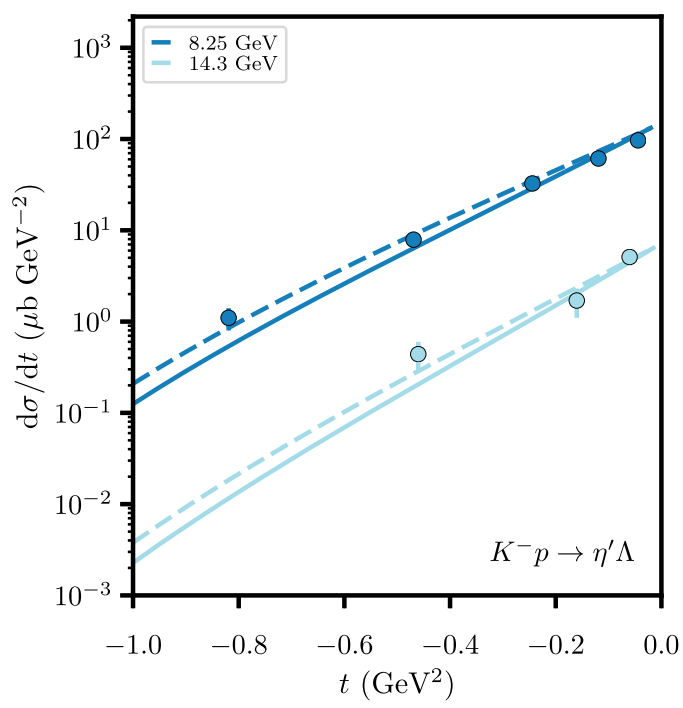

(b) $K^{-} p \rightarrow \eta^{\prime} \Lambda$

FIG. 12. Results for the strangeness exchange $\eta^{(\prime)} \Lambda$ production channels. Scaling and conventions are as in Fig. 4 .

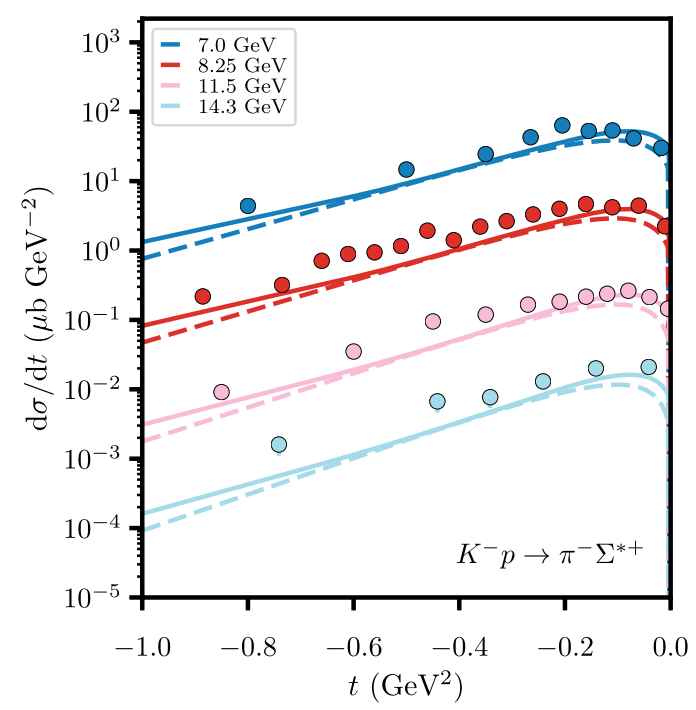

(a) $K^{-} p \rightarrow \pi^{-} \Sigma^{*+}$

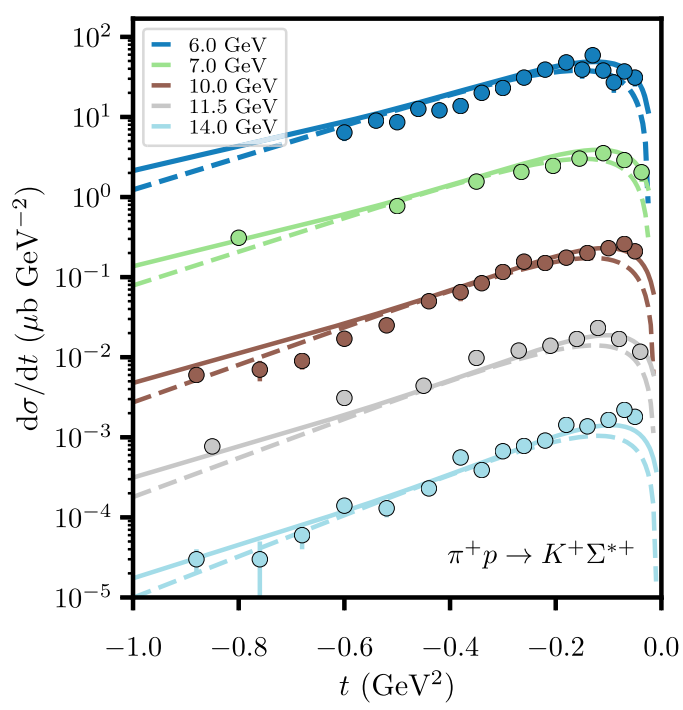

(b) $\pi^{+} p \rightarrow K^{+} \Sigma^{*+}$

FIG. 13. Results for the strangeness exchange $\pi \Sigma^{*}$ and $K \Sigma^{*}$ production channels. Scaling and conventions are as in Fig. 4.

undiscovered $\rho_{2}\left(I^{G} J^{P C}=1^{+} 2^{--}\right)[34,37] .^{8}$ Due to its positive signature, this contribution is not required to vanish at $\alpha_{\rho_{2}}(t)=0$ and might be even more important at forward angles than the $b_{1}$ exchange, provided that $\alpha_{\rho_{2}}(t)$ is similar to $\alpha_{b_{1}}(t)$; note, however, that $\alpha_{\rho_{2}}(t)$ is

\footnotetext{
${ }^{8}$ These quantum numbers are not exotic (only the $0^{--}$is), and both the quark model and lattice QCD results predict the existence of such states $[83,84]$. There are some experimental indications of the existence of $\rho_{2}$ and $\omega_{2}$ mesons $[85,86]$. However, these states have been observed by a single group and are poorly established, thus needing confirmation [38].
}

undetermined, as pointed out in [12]. This is because the NWSZ of the $b_{1}$ lies at $t=0$, which forces the $b_{1}$ contribution to vanish in the forward direction, independent of the factors in Eq. (5). This lack of strength in our model in the forward direction hinders an unambiguous extraction of the $b_{1}$ couplings. While we do include a contribution from the $b_{1}$ exchange to absorb the different energy dependence at lower energies, we do not quote the results for the latter since these couplings are unreliable.

For the strangeness exchange channels, the fits are somewhat more difficult since one cannot separate the $K^{*}$ from $K_{2}^{*}$ exchanges due to the lack of definite $G$ parity. 
TABLE IV. Couplings $\hat{\beta}_{\mu_{i} \mu_{f}}^{\text {eif }}\left(t=m_{e}^{2}\right)$ from the SU(3)-EXD fits. Subscripts denote helicities. The fixed residues are indicated with an asterisk. The exponential factors are not included to extrapolate to the pole. A global fit yields $b_{\mathrm{nf}}=1.31 \mathrm{GeV}^{-2}$ and $b_{\text {sf }}=0.54 \mathrm{GeV}^{-2}$.

\begin{tabular}{|c|c|c|c|c|}
\hline if & $\rho$ & $a_{2}$ & $K^{*}$ & $K_{2}^{*}$ \\
\hline$\pi^{-} \pi^{-}$ & $8.40^{*}$ & . & . & . \\
\hline$K^{+} K^{+}$ & $-4.20^{*}$ & $4.20^{*}$ & . & . \\
\hline$\pi^{0} \eta$ & . & 5.78 & . & . \\
\hline$\pi^{0} \eta^{\prime}$ & . & 6.10 & $\cdot$ & $\cdot$ \\
\hline$K^{-} \pi^{-}$ & . & $\cdot$ & $-5.94^{*}$ & $5.94^{*}$ \\
\hline$\overline{K^{0}} \eta$ & . & . & -7.20 & -3.48 \\
\hline$\overline{K^{0}} \eta^{\prime}$ & $\cdot$ & . & 1.04 & 7.13 \\
\hline$\pi^{0} \omega_{0}$ & $0^{*}$ & . & $\cdot$ & $\cdot$ \\
\hline$\pi^{0} \omega_{+}$ & -15.88 & . & . & . \\
\hline$p_{+} p_{+}$ & 1.06 & 1.06 & . & . \\
\hline$p_{-} p_{+}$ & 5.63 & 5.63 & $\cdot$ & $\cdot$ \\
\hline$n_{+} \Lambda_{+}$ & $\cdot$ & $\cdot$ & -3.33 & -3.33 \\
\hline$n_{-} \Lambda_{+}$ & . & . & -5.02 & -5.02 \\
\hline$p_{+} \Sigma_{+}^{+}$ & . & . & -5.16 & -5.16 \\
\hline$p_{-} \Sigma_{+}^{+}$ & $\cdot$ & $\cdot$ & 2.19 & 2.19 \\
\hline$p_{-} \Delta_{+\frac{1}{2}}^{+}$ & 4.08 & 4.08 & . & $\cdot$ \\
\hline$p_{+} \Delta_{+\frac{3}{2}}^{+}$ & 9.27 & 9.27 & . & . \\
\hline$p_{+} \Delta_{+\frac{1}{2}}^{+}$ & 1.70 & 1.70 & . & . \\
\hline$p_{-} \Delta_{+\frac{3}{2}}^{+}$ & $0^{*}$ & $0^{*}$ & . & . \\
\hline$p_{-} \Sigma_{+\frac{1}{2}}^{*+}$ & . & . & -2.39 & -2.39 \\
\hline$p_{+} \Sigma_{+\frac{3}{2}}^{*+}$ & . & $\cdot$ & -6.12 & -6.12 \\
\hline$p_{+} \Sigma_{+\frac{1}{2}}^{*+}$ & . & . & -1.88 & -1.88 \\
\hline$p \_\Sigma_{+\frac{3}{2}}^{*+}$ & . & . & $0^{*}$ & $0^{*}$ \\
\hline
\end{tabular}

Additionally, there is less sensitivity to the trajectory parameters due to a limited energy range in the data. We therefore keep the trajectories fixed to the ones used in the global SU(3)-EXD fit. Additionally, the EXD constraint is imposed on the fits to reduce the number of free parameters. In the global SU(3)-EXD fits shown in Figs. 10a-11c and Table VII, it appears that the $0^{-\frac{1}{2}+}$ production channels are dominated by Regge-pole nonflip contributions in the domain $0 \leq-t \leq 0.25 \mathrm{GeV}^{2}$. We can therefore carry out two-step fits, where we first determine the nonflip coupling from these very forward data. In a second step, we fix the nonflip coupling and extract the flip contribution from a full $t$ range fit. One observes that the helicity-flip couplings do not obey the SU(3) constraints well, in contrast to the nonflip contributions. Obtaining the helicity flip contributions from the fit turns out to be ambiguous. They depend strongly on the considered $t$ range, and the final results deviate heavily from the SU(3)-EXD predictions. This issue was anticipated in the previous section, where we
TABLE V. Couplings $g_{\mu_{i} \mu_{f}}^{e i f}$ from the unconstrained fits. Subscripts denote helicities. Residues that have been kept fixed in the fit are denoted by an asterisk.

\begin{tabular}{|c|c|c|c|c|}
\hline if & $\rho$ & $a_{2}$ & $K^{*}$ & $K_{2}^{*}$ \\
\hline$\pi^{-} \pi^{-}$ & $8.40^{*}$ & $\cdot$ & . & . \\
\hline$K^{+} K^{+}$ & -3.93 & 3.93 & . & . \\
\hline$\pi^{0} \eta$ & . & 5.43 & . & . \\
\hline$\pi^{0} \eta^{\prime}$ & . & 3.93 & . & $\cdot$ \\
\hline$K^{-} \pi^{-}$ & . & $\cdot$ & $-5.94^{*}$ & $5.94^{*}$ \\
\hline$\overline{K^{0}} \eta$ & . & . & -7.17 & -2.35 \\
\hline$\overline{K^{0}} \eta^{\prime}$ & . & . & 0 & 6.34 \\
\hline$\pi^{0} \omega_{0}$ & $0^{*}$ & . & . & . \\
\hline$\pi^{0} \omega_{+}$ & -9.46 & $\cdot$ & . & . \\
\hline$p_{+} p_{+}$ & 1.76 & 1.43 & . & . \\
\hline$p_{-} p_{+}$ & 8.02 & 7.59 & $\cdot$ & $\cdot$ \\
\hline$n_{+} \Lambda_{+}$ & $\cdot$ & $\cdot$ & -3.77 & -3.77 \\
\hline$n_{-} \Lambda_{+}$ & . & . & -4.31 & -4.31 \\
\hline$p_{+} \Sigma_{+}^{+}$ & . & . & -5.05 & -5.05 \\
\hline$p_{-} \Sigma_{+}^{+}$ & . & . & 2.79 & 2.79 \\
\hline$p_{-} \Delta_{+\frac{1}{2}}^{+}$ & 6.26 & 3.22 & . & . \\
\hline$p_{+} \Delta_{+\frac{3}{2}}^{+}$ & 14.23 & 7.32 & . & . \\
\hline$p_{+} \Delta_{+\frac{1}{2}}^{+}$ & 1.25 & -1.83 & . & . \\
\hline$p_{-} \Delta_{+\frac{3}{2}}^{+}$ & $0^{*}$ & $0^{*}$ & . & . \\
\hline$p \_\Sigma_{+\frac{1}{2}}^{*+}$ & . & . & $-2.39^{*}$ & $-2.39 *$ \\
\hline$p_{+} \Sigma_{+\frac{3}{2}}^{*+}$ & . & . & -10.00 & -6.12 \\
\hline$p_{+} \Sigma_{+\frac{1}{2}}^{*+}$ & . & . & 0 & -0.94 \\
\hline$p \_\Sigma_{+\frac{3}{2}}^{*+}$ & . & . & $0^{*}$ & $0^{*}$ \\
\hline
\end{tabular}

commented (based on the $s$-dependence) that the large $-t$ behavior of the cross section is dominated by contributions other than the Regge poles considered here.

The $\hat{\beta}^{K_{(2)}^{*} K \eta^{(1)}}$ couplings are determined in a separate fit using the $\eta^{(\prime)} \Lambda$ production data in Figs. 12a and 12b. As mentioned before, the SU(3) constraint does not hold well for these couplings.

For the $\Sigma^{*}$ production channels in Figs. 13a and 13b, we fix the $\hat{\beta}_{+\frac{1}{2}+\frac{1}{2}}^{K_{2}^{*} N \Sigma^{*}}$ coupling constants and the exponential $t$-dependence to the ones obtained in the global SU(3)EXD fit. EXD is not imposed for the remaining coupling constants. The SU(3)-EXD fit already provided a reliable representation of these channels. The main difference to the unconstrained fit is the forward behavior of the cross section. Indeed, as discussed before, the single-meson exchange approximation forces the nonflip component to vanish at $t=0$. Therefore, no amplitude survives in the forward direction. In the unconstrained fit, the nonflip component is allowed to contribute in the forward direction. This feature seems to be favored by the data. 
TABLE VI. Residue exponential factors $b_{\mu_{i} \mu_{f}}^{\text {eif }}$ from the unconstrained fits. Subscripts denote helicities. The fits are only sensitive to the product of the top and bottom residues.

\begin{tabular}{|c|c|c|}
\hline$e 13$ & $e 24$ & $b_{\mu_{1} \mu_{3}}^{e 13}+b_{\mu_{2} \mu_{4}}^{e 24}\left(\mathrm{GeV}^{-2}\right)$ \\
\hline$\rho \pi \pi$ & $\rho N_{+} N_{+}$ & 0 \\
\hline$\rho \pi \pi$ & $\rho N_{-} N_{+}$ & 0.86 \\
\hline$a_{2} \pi \eta^{(\prime)}$ & $a_{2} N_{+} N_{+}$ & 0 \\
\hline$a_{2} \pi \eta^{(\prime)}$ & $a_{2} N_{-} N_{+}$ & 0.27 \\
\hline$\rho K K$ & $\rho N_{+} N_{+}$ & -0.55 \\
\hline$\rho K K$ & $\rho N_{-} N_{+}$ & 0.32 \\
\hline$a_{2} K K$ & $a_{2} N_{+} N_{+}$ & 0.01 \\
\hline$a_{2} K K$ & $a_{2} N_{-} N_{+}$ & 0.28 \\
\hline$\rho \pi \omega_{+1}$ & $\rho N_{+} N_{+}$ & 0.82 \\
\hline$\rho \pi \omega_{+1}$ & $\rho N_{-} N_{+}$ & 1.68 \\
\hline$\rho \pi \pi$ & $\rho N_{+} \Delta_{-\frac{1}{2}}$ & 1.38 \\
\hline$\rho \pi \pi$ & $\rho N_{+} \Delta_{+\frac{3}{2}}$ & 1.38 \\
\hline$\rho \pi \pi$ & $\rho N_{+} \Delta_{+\frac{1}{2}}$ & 1.85 \\
\hline$a_{2} \pi \eta^{(\prime)}$ & $a_{2} N_{+} \Delta_{-\frac{1}{2}}$ & -0.16 \\
\hline$a_{2} \pi \eta^{(\prime)}$ & $a_{2} N_{+} \Delta_{+\frac{3}{2}}$ & -0.16 \\
\hline$a_{2} \pi \eta^{(\prime)}$ & $a_{2} N_{+} \Delta_{+\frac{1}{2}}$ & 1.30 \\
\hline$\rho K K$ & $\rho N_{+} \Delta_{-\frac{1}{2}}$ & 0.83 \\
\hline$\rho K K$ & $\rho N_{+} \Delta_{+\frac{3}{2}}$ & 0.83 \\
\hline$\rho K K$ & $\rho N_{+} \Delta_{+\frac{1}{2}}$ & 1.31 \\
\hline$a_{2} K K$ & $a_{2} N_{+} \Delta_{-\frac{1}{2}}$ & -0.15 \\
\hline$a_{2} K K$ & $a_{2} N_{+} \Delta_{+\frac{3}{2}}$ & -0.15 \\
\hline$a_{2} K K$ & $a_{2} N_{+} \Delta_{+\frac{1}{2}}$ & 1.31 \\
\hline$K_{(2)}^{*} K \pi$ & $K_{(2)}^{*} N_{+} \Sigma_{+}$ & 1.26 \\
\hline$K_{(2)}^{*} K \pi$ & $K_{(2)}^{*} N_{+} \Sigma_{-}$ & 0.54 \\
\hline$K_{(2)}^{*} K \pi$ & $K_{(2)}^{*} N_{+} \Lambda_{+}$ & 1.31 \\
\hline$K_{(2)}^{*} K \pi$ & $K_{(2)}^{*} N_{+} \Lambda_{-}$ & 0.54 \\
\hline$K_{(2)}^{*} K \eta^{(\prime)}$ & $K_{(2)}^{*} N_{+} \Lambda_{+}$ & 0 \\
\hline$K_{(2)}^{*} K \eta^{(\prime)}$ & $K_{(2)}^{*} N_{+} \Lambda_{+}$ & 0 \\
\hline$K_{(2)}^{*} K \pi$ & $K_{(2)}^{*} N_{+} \Sigma_{-\frac{1}{2}}^{*}$ & 0.54 \\
\hline$K_{(2)}^{*} K \pi$ & $K_{(2)}^{*} N_{+} \Sigma_{+\frac{3}{2}}^{*}$ & 0.54 \\
\hline$K_{(2)}^{*} K \pi$ & $K_{(2)}^{*} N_{+} \Sigma_{+\frac{1}{2}}^{*}$ & 1.31 \\
\hline
\end{tabular}

\section{CONCLUSIONS}

We assessed the applicability of the Regge pole model by performing a global fit to charge and strange exchange quasi-two-body reactions at large momenta $p_{\text {lab }} \geq 5 \mathrm{GeV}$. We have found that the Regge pole model provides a good description of the data for a large amount of channels, while requiring only a small number of free SU(3) and EXD related parameters. It was shown that the inclusion of these constraints offers a solid way to reduce the number of free parameters of the fit. The large number of free parameters in the unconstrained fit allows for too much freedom compared with the number of available data to yield a unique result. SU(3) and EXD constraints are especially useful to determine the relevant regions of the vast parameter space of the residues. In kinematic domains where wrong-signature zeros can be expected (such as in channels dominated by $\rho$ exchange), secondary contributions become relevant. For these channels, we find the single pole model and factorization to work well in the domain $0 \leq-t \leq 0.5 \mathrm{GeV}^{2}$. The cross sections of reactions dominated by $a_{2}$ exchanges are well reproduced by the Regge pole models up to $t \sim-0.8 \mathrm{GeV}^{2}$, due to the lack of a dip. Reactions dominated by strangeness exchanges follow the Regge pole model remarkably well in the forward region up to $t \sim-0.6 \mathrm{GeV}^{2}$. Especially those channels dominated by nonflip baryon vertices are in good agreement with the constraints imposed by SU(3), which should therefore be considered in future fits. These are the kinematic domains where factorization can be used as a reliable approximation to model beam-target fragmentation. The presented model provides a solid description of the production mechanism needed to describe the production amplitude in peripheral resonance production of relevance to hybrid searches. Our predictions and our model will be made available online on the JPAC website $[87,88]$. With the online version of the model, users have the possibility to vary the model parameters and generate the observables.

\section{ACKNOWLEDGMENTS}

We dedicate this work to the memory of Mike Pennington. He was an example of humanity and scientific dedication whose efforts and support made the JPAC Collaboration possible. This work was supported by Research Foundation-Flanders (FWO), the U.S. Department of Energy under Grants No. DE-AC0506OR23177 and No. DE-FG02-87ER40365, U.S. National Science Foundation under Award numbers PHY-1415459, PHY-1205019, and PHY-1513524, PAPIIT-DGAPA (UNAM, Mexico) Grant No. IA101717, CONACYT (Mexico) Grant No. 251817.

\section{APPENDIX A: KINEMATICS AND CONVENTIONS}

In the $s$-channel center-of-mass (c.m.) frame, the cosine of the scattering angle for $1+2 \rightarrow 3+4$ is given by

$$
\begin{array}{r}
z_{s}=\frac{s^{2}+s\left(2 t-\sum_{i} m_{i}^{2}\right)+\left(m_{1}^{2}-m_{2}^{2}\right)\left(m_{3}^{2}-m_{4}^{2}\right)}{S_{12}(s) S_{34}(s)}, \\
S_{i j}^{2}(s) \equiv\left[s-\left(m_{i}+m_{j}\right)^{2}\right]\left[s-\left(m_{i}-m_{j}\right)^{2}\right] .
\end{array}
$$

The conventions are illustrated in Fig. 2. Introducing $t_{\min }(s) \equiv t\left(z_{s}=+1\right)$ and $t^{\prime}=t-t_{\min }$, the above can be simplified as 


$$
z_{s}=1+\frac{2 s t^{\prime}}{S_{12}(s) S_{34}(s)}
$$

or in other words, $1-z_{s} \sim-t^{\prime}$. At leading $s, t=t^{\prime}$. Furthermore,

$$
\begin{gathered}
E_{i}=\frac{s+m_{i}^{2}-m_{j}^{2}}{2 \sqrt{s}}, \\
\left|\vec{p}_{i}\right|=\frac{S_{i j}(s)}{2 \sqrt{s}},
\end{gathered}
$$

where $(i, j)$ is an $s$-channel pair.

The expressions for the helicity amplitudes in terms of the covariant couplings are obtained by properly selecting the polarization angles. We use the "particle 2" convention of Jacob and Wick [89], which requires that for the helicity states of the two particles in a two-particle $s$-channel helicity state

$$
\lim _{\vec{p} \rightarrow 0}\langle-\vec{p},-\mu \mid \vec{p}, \mu\rangle=1
$$

Since the baryons in our convention are "particle 2 " in the $s$-channel helicity pairs, one must use the following conventions:

$$
u^{(2)}(p, \mu)=\sqrt{E+m}\left(\begin{array}{c}
1 \\
\frac{\vec{p} \cdot \vec{\sigma}}{E+m}
\end{array}\right) \chi_{\mu}^{(2)}(\theta, \phi)
$$

where $\chi^{(2)}(\theta, \phi)$ is a "particle 2 " Pauli spinor, i.e., the fermion (in a two-particle pair) goes opposite to the direction determined by $(\theta, \phi)$.

For a "particle 1" Pauli spinor, we have

$$
\begin{gathered}
\chi_{+\frac{1}{2}}^{(1)}(\theta, \phi)=\left(\begin{array}{c}
\cos \theta \\
e^{i \phi} \sin \theta
\end{array}\right), \\
\chi_{-\frac{1}{2}}^{(1)}(\theta, \phi)=\left(\begin{array}{c}
-e^{-i \phi} \sin \theta \\
\cos \theta
\end{array}\right) .
\end{gathered}
$$

Using the above definition, the "particle 2" Pauli spinor is defined as

$$
\chi_{-\mu}^{(2)}(\pi-\theta, \pi+\phi)=\chi_{\mu}^{(1)}(\theta, \phi) .
$$

Also, we consider $\phi=0$ from hereon. The rotation $\vec{p} \rightarrow$ $-\vec{p}$ then corresponds to $(\theta, \phi=0) \rightarrow(\pi+\theta, \phi=0)$. For the produced massive vector meson, we use the polarization vectors

$$
\begin{gathered}
\epsilon^{\nu}(p, \mu= \pm 1)=\frac{1}{\sqrt{2}}(0,-\mu \cos \theta,-i, \mu \sin \theta)^{T} \\
\epsilon^{\nu}(p, \mu=0)=\frac{1}{m}\left(|\vec{p}|, p^{0} \vec{e}_{\vec{p}}\right)^{T},
\end{gathered}
$$

where $\vec{e}_{\vec{p}}$ is the unit vector in the direction of $\vec{p}$. For a "particle 2 " vector, one finds

$$
\epsilon^{(2) \nu}(p, \mu)=-g^{\nu \nu} \epsilon^{\nu}(\tilde{p},-\mu)
$$

where the index $\nu$ is not summed over. For the spin-3/2 Rarita-Schwinger spinor, we use the expression

$$
u^{(1) \nu}(p, \mu)=\sum_{\mu_{1}, \mu_{2}}\left\langle 1, \mu_{1} ; \frac{1}{2} \mu_{2} \mid \frac{3}{2} \mu\right\rangle \epsilon^{\nu}\left(p, \mu_{1}\right) u^{(1)}\left(p, \mu_{2}\right)
$$

since $\vec{p}$ lies in the $x-z$ scattering plane. Here, $\tilde{p}=\left(p^{0}\right.$, $-\vec{p})$, and $-\vec{p}$ has spherical angles $(\pi-\theta, \phi+\pi)$. For a "particle 2" spin-3/2 spinor, we use the same form, with the spinor and polarization vector substituted by their "particle 2 " form. In the following, we drop the explicit "particle 1" and "particle 2" reference for brevity of notation. The spin-3/2 spinors satisfy the Rarita-Schwinger equations

$$
\begin{gathered}
p_{\nu} u^{\nu}(p, \mu)=0, \\
\gamma_{\nu} u^{\nu}(p, \mu)=0, \\
(\not p-m) u^{\nu}(p, \mu)=0 .
\end{gathered}
$$

Within our conventions, parity invariance implies

$$
\begin{gathered}
A_{\mu_{4} \mu_{3} \mu_{2} \mu_{1}}=\eta^{s} A_{-\mu_{4}-\mu_{3}-\mu_{2}-\mu_{1}}, \\
\eta^{s}=\frac{\eta_{1} \eta_{2}}{\eta_{3} \eta_{4}}(-1)^{\mu^{\prime}-\mu}
\end{gathered}
$$

where $\eta_{i}=P_{i}(-1)^{s_{i}}$ is the naturality of a particle $i$ with parity $P_{i}$ and spin $s_{i}$. For the individual vertices, one has

$$
\begin{aligned}
& \beta_{\mu_{3} \mu_{1}}=\eta_{e} P_{1} P_{3}(-1)^{s_{3}-s_{1}}(-1)^{\mu_{3}-\mu_{1}} \beta_{-\mu_{3}-\mu_{1}}, \\
& \beta_{\mu_{4} \mu_{2}}=\eta_{e} P_{2} P_{4}(-1)^{s_{4}-s_{2}}(-1)^{\mu_{2}-\mu_{4}} \beta_{-\mu_{4}-\mu_{2}},
\end{aligned}
$$

where $\eta_{e}$ is the naturality of the exchange.

\section{APPENDIX B: FACTORIZATION of REGGE POLE RESIDUES}

Since testing factorization is the central topic of this work, we derive its implications on helicity amplitudes. The kinematic $t$-singularities in the $s$-channel partial waves are given by the half-angle factors in Eq. (7). Hence, for $z_{s} \rightarrow+1$ (or equivalently $t^{\prime} \rightarrow 0$ ), one finds

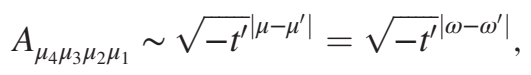

where $\omega=\mu_{1}-\mu_{3}$ and $\omega^{\prime}=\mu_{2}-\mu_{4}$. The above is the most singular kinematic behavior of the amplitude and cannot be cast into the factorizable form in Eq. (3). One can show [90] that the simplest factorizable form consistent with a definite parity exchange is given by 


$$
A_{\mu_{4} \mu_{3} \mu_{2} \mu_{1}} \sim \sqrt{-t}^{|\omega|+\left|\omega^{\prime}\right|} .
$$

In Eq. (11), $t^{\prime}$ is used explicitly for the factors stemming from the half-angle factors in Eq. (B1).

\section{APPENDIX C: INETERACTION LANGRANGIANS}

In order to relate the helicity couplings to those of modern literature, we start from the set of effective Lagrangians given below. We consider interactions of pseudoscalars $(\mathrm{P})$, vectors $(\mathrm{V})$, axial vectors $(\mathrm{A})$, tensors (T), octet baryons (B), and decuplet baryons (D).

We use the following conventions in the global SU(3) fit. The explicit form of the matrices for pseudoscalar, vector, and tensor mesons is

$$
\begin{gathered}
P=\left(\begin{array}{ccc}
\frac{\pi^{0}}{\sqrt{2}}+\frac{\eta^{8}}{\sqrt{6}}+\frac{\eta^{1}}{\sqrt{3}} & \pi^{+} & K^{+} \\
\pi^{-} & \frac{-\pi^{0}}{\sqrt{2}}+\frac{\eta^{8}}{\sqrt{6}}+\frac{\eta^{1}}{\sqrt{3}} & K^{0} \\
K^{-} & \bar{K}^{0} & -\sqrt{\frac{2}{3} \eta^{8}+\frac{\eta^{1}}{\sqrt{3}}}
\end{array}\right), \\
\eta^{1}=\eta^{\prime} \cos \theta_{P}-\eta \sin \theta_{P}, \\
\eta^{8}=\eta \cos \theta_{P}+\eta^{\prime} \sin \theta_{P}, \\
V^{8}=\left(\begin{array}{ccc}
\frac{1}{\sqrt{2}} \rho^{0}+\frac{1}{\sqrt{6}} \omega^{8} & \rho^{+} & K^{*+} \\
\rho^{-} & -\frac{1}{\sqrt{2}} \rho^{0}+\frac{1}{\sqrt{6}} \omega^{8} & K^{* 0} \\
K^{*-} & \bar{K}^{* 0} & -\frac{2}{\sqrt{6}} \omega^{8}
\end{array}\right), \\
T^{1}= \\
T_{2}^{1}=\left(\begin{array}{ccc}
\frac{a_{2}}{\sqrt{2}}+\frac{f_{2}^{8}}{\sqrt{6}} & a_{2}^{+} & K_{2}^{*+} \\
a_{2}^{-} & -\frac{a_{2}^{0}}{\sqrt{2}}+\frac{f_{2}^{8}}{\sqrt{6}} & K_{2}^{* 0} \\
K_{2}^{*-} & \bar{K}_{2}^{* 0} & -2 \frac{f_{2}^{8}}{\sqrt{6}}
\end{array}\right), \\
f_{2}^{8}=f_{2} \sin \theta_{T}+f_{2}^{\prime} \cos \theta_{T} .
\end{gathered}
$$

Here, $\theta_{T}$ and $\theta_{P}$ are the tensor and pseudoscalar mixing angles, respectively, between the singlet and octet. Furthermore, we call $\theta_{P}^{I}$ the ideal mixing angle with $\sin \theta_{P}^{I}=1 / \sqrt{3}$ and $\cos \theta_{P}^{I}=\sqrt{2 / 3}$. In the case of ideal mixing for vector mesons, the following relations hold:

$$
\omega=\frac{1}{\sqrt{3}} \omega^{8}+\sqrt{\frac{2}{3}} \omega^{1}, \quad \phi=\sqrt{\frac{2}{3}} \omega^{8}-\frac{1}{\sqrt{3}} \omega^{1} .
$$

Note that we have implicitly assumed a nonet symmetry for the pseudoscalar mesons in Eq. (C1), where the singlet/ octet coupling ratio is $S_{T}=\sqrt{2}$ [33]. The latter corresponds to neglecting the coupling to the $s \bar{s}$ content. In the following, the trace is taken over flavor space, corresponding to the isospin couplings of the different channels in $\mathrm{SU}(3)$ symmetry.

The three-meson interaction Lagrangians consist only of a symmetric coupling due to $G$-parity conservation. All the couplings appearing in the following are fitting parameters, obtaining values as explained in Appendix D. The Lagrangians describing the couplings of tensor mesons to pseudoscalar and vector mesons are given by [91-93]

$$
\mathcal{L}_{T P P}=c_{T P P}^{8}\left\langle\mathcal{T}_{\mu \nu}^{8} \Theta_{P}^{\mu \nu}\right\rangle+\frac{c_{T P P}^{1}}{\sqrt{3}} \mathcal{T}_{\mu \nu}^{1}\left\langle\Theta_{P}^{\mu \nu}\right\rangle
$$

\begin{tabular}{|c|c|}
\hline$\hat{\beta}_{\mu_{i} \mu_{f}}^{\mathrm{Rif}}(t)$ & Expression \\
\hline$\hat{\beta}_{00}^{\mathrm{VPP}}$ & $\sqrt{2} g_{\mathrm{VPP}}$ \\
\hline$\hat{\beta}_{+\frac{1}{2}+\frac{1}{2}}^{\mathrm{VBB}}$ & $\sqrt{2} g_{\mathrm{VBB}}^{v}$ \\
\hline$\hat{\beta}_{-\frac{1}{2}+\frac{1}{2}}^{\mathrm{VBB}}$ & $\sqrt{2} g_{\mathrm{VBB}}^{t} /\left(m_{2}+m_{4}\right)$ \\
\hline$\hat{\beta}_{00}^{T P P}$ & $g_{T P P} / 2$ \\
\hline$\hat{\beta}_{+\frac{1}{2}+\frac{1}{2}}^{T B B}$ & $-\frac{2}{m_{2}+m_{4}}\left(g_{T B B}^{(2)}+g_{T B B}^{(1)}\right)$ \\
\hline$\hat{\beta}_{-\frac{1}{2}+\frac{1}{2}}^{T B B}$ & $2 g_{T B B}^{(2)} /\left(m_{2}+m_{4}\right)^{2}$ \\
\hline$\hat{\beta}_{-\frac{1}{2}+\frac{1}{2}}^{\mathrm{VBD}}$ & $2 \frac{g_{\mathrm{VBD}}^{(1)} m_{2}\left(m_{2}+m_{4}\right)-g_{\mathrm{VBD}}^{(2)}\left(2 t-m_{4}\left(m_{2}-m_{4}\right)\right)+2 t g_{\mathrm{VBD}}^{(3)}}{\sqrt{3} m_{4}\left(m_{2}+m_{4}\right)^{2}}$ \\
\hline$\hat{\beta}_{+\frac{1}{2}+\frac{3}{2}}^{\mathrm{VBD}}$ & $\frac{2}{\left(m_{2}+m_{4}\right)^{2}}\left[g_{\mathrm{VBD}}^{(1)}\left(m_{2}+m_{4}\right)-g_{\mathrm{VBD}}^{(2)}\left(m_{2}-m_{4}\right)\right]$ \\
\hline$\hat{\beta}_{+\frac{1}{2}+\frac{1}{2}}^{\mathrm{VBD}}$ & $\frac{g_{\mathrm{VBD}}^{(1)}\left(m_{2}+m_{4}\right)-g_{\mathrm{VDD}}^{(2)}\left(m_{2}-m_{4}\right)-g_{\mathrm{VBD}}^{(3)}\left(m_{2}-m_{4}\right)}{\sqrt{12} m_{4}\left(m_{2}+m_{4}\right)}(-t)$ \\
\hline$\hat{\beta}_{-\frac{1}{2}+\frac{3}{2}}^{\mathrm{VBD}}$ & $2 g_{\mathrm{VBD}}^{(2)} /\left(m_{2}+m_{4}\right)^{2}$ \\
\hline$\hat{\beta}_{00}^{V P V}$ & 0 (parity) \\
\hline$\hat{\beta}_{0+1}^{V P V}$ & $g_{V V P} / 2$ \\
\hline$\hat{\beta}_{0,0}^{A V P}(t)$ & $g_{A V P} /\left(\sqrt{2} m_{3}\right)$ \\
\hline$\hat{\beta}_{0+1}^{A V P}(t)$ & $g_{A V P}^{\prime}$ \\
\hline$\hat{\beta}_{+\frac{1}{2}+\frac{1}{2}}^{A B B}$ & $0\left(\right.$ for $\left.C_{A}=-1\right)$ \\
\hline$\hat{\beta}_{-\frac{1}{2}+\frac{1}{2}}^{A B B}$ & $\sqrt{2} g_{A B B}^{t} /\left(m_{2}+m_{4}\right)$ \\
\hline$\hat{\beta}_{00}^{T P V}$ & 0 (parity) \\
\hline$\underline{\hat{\beta}_{0+1}^{T P V}}$ & $g_{T P V} / \sqrt{2}$ \\
\hline
\end{tabular}

TABLE VII. Listing of the reduced $s$-channel residues and their expressions in the single-particle exchange formalism. The expressions are given up to the flavor trace. We assumed $M_{S}=\left(m_{i}+m_{f}\right) / 2$. In practice, these couplings are evaluated on the mass pole of the exchanged particle. Only the lowest order $t$-dependence, which is compatible with factorization, is used in the residue. Every residue contains an additional $\sqrt{s_{0}} J_{e}$, where $J_{e}$ is the spin of the lightest particle on the trajectory. 


$$
\mathcal{L}_{T V P}=g_{T V P}\left\langle\mathcal{T}^{[\mu \nu] \alpha}\left[\tilde{V}_{\mu \nu}, \partial_{\alpha} P\right]\right\rangle,
$$

where

$$
\begin{gathered}
\Theta_{P}^{\mu \nu}=\partial^{\mu} P \partial^{\nu} P-g^{\mu \nu}(\partial \cdot P)^{2}, \\
\mathcal{T}^{[\mu \nu] \alpha}=\partial^{\mu} \mathcal{T}^{\nu \alpha}-\partial^{\nu} \mathcal{T}^{\mu \alpha}, \\
\mathcal{T}_{\mu \nu}=\mathcal{T}_{\mu \nu}^{8}+\frac{\mathcal{T}_{\mu \nu}^{1}}{\sqrt{3}}, \\
\tilde{V}_{\mu \nu}=\frac{1}{2} \epsilon_{\mu \nu \rho \sigma} V^{\rho \sigma}, \\
V^{\mu \nu}=\partial^{\mu} V^{\nu}-\partial^{\nu} V^{\mu} .
\end{gathered}
$$

Concerning the couplings of vector to pseudoscalar mesons, the Lagrangians read

$$
\begin{gathered}
\mathcal{L}_{\mathrm{VPP}}=-\mathrm{i} g_{\mathrm{VPP}}\left\langle\left[P, \partial_{\mu} P\right] V^{\mu}\right\rangle, \\
\mathcal{L}_{V V P}=\frac{g_{V V P}}{2} \epsilon_{\mu \nu \alpha \beta}\left\langle\left\{\partial^{\mu} V^{\nu}, \partial^{\alpha} V^{\beta}\right\} P\right\rangle .
\end{gathered}
$$

The Lagrangian that couples vector, pseudoscalar, and axial mesons $b_{1}$ is given by (we do not consider SU(3) relations for the $b_{1}$ exchange since it is the only axial exchange considered here) $[94,95]$.

$$
\mathcal{L}_{V P A}=g_{V P A} A_{\mu} V^{\mu} P+g_{V P A}^{\prime} A_{\mu \nu} V^{\mu \nu} P .
$$

Since one can couple two octets to both a symmetric and an antisymmetric octet, each meson $-B-B$ vertex must be decomposed into two irreducible structures, with independent couplings. The couplings of the vector-meson fields $V_{\mu}$ with momentum $q$ to the octet baryons are described by the following Lagrangian [96]:

$$
\begin{aligned}
\mathcal{L}_{\mathrm{VBB}}= & \left\langle\overline { B } \left[\left(g_{\mathrm{VBB}}^{v, F}\left[V_{\mu}^{8}, B\right]+g_{\mathrm{VBB}}^{v, D}\left\{V_{\mu}^{8}, B\right\}+g_{\mathrm{VBB}}^{v, S} V_{\mu}^{1} B\right) \gamma^{\mu}\right.\right. \\
& +\left(g_{\mathrm{VBB}}^{t, F}\left[V_{\mu}^{8}, B\right]+g_{\mathrm{VBB}}^{t, D}\left\{V_{\mu}^{8}, B\right\}\right. \\
& \left.\left.\left.+g_{\mathrm{VBB}}^{t, S} V_{\mu}^{1} B\right) \frac{\mathrm{i} \sigma^{\mu \nu} q_{\nu}}{2 M_{S}}\right]\right\rangle,
\end{aligned}
$$

where $M_{S}=\left(m_{B 1}+m_{B 2}\right) / 2$, and $m_{B 1}$ and $m_{B 2}$ are the masses of the incoming and the outgoing baryon, respectively. The octet-baryon matrix is explicitly given as

$$
B=\left(\begin{array}{ccc}
\frac{1}{\sqrt{2}} \Sigma^{0}+\frac{1}{\sqrt{6}} \Lambda & \Sigma^{+} & p \\
\Sigma^{-} & -\frac{1}{\sqrt{2}} \Sigma^{0}+\frac{1}{\sqrt{6}} \Lambda & n \\
\Xi^{-} & \Xi^{0} & -\frac{2}{\sqrt{6}} \Lambda
\end{array}\right) .
$$

In the following, for simplicity we drop the notation with traces over commutators/anticommutators in flavor space. The couplings of tensor mesons $f^{\mu \nu}$ to the baryons are given in Refs. [97-101]. We follow the general definitions of the Lagrangian

$$
\mathcal{L}_{T B B}=\mathrm{i} \frac{g_{T B B}^{(1)}}{4 M_{S}} \bar{B}\left(\gamma_{\mu} \stackrel{\leftrightarrow}{\partial_{\nu}}+\gamma_{\nu} \stackrel{\leftrightarrow}{\partial_{\mu}}\right) B f^{\mu \nu}+\frac{g_{T B B}^{(2)}}{M_{S}^{2}} \partial_{\mu} \bar{B} \partial_{\nu} B f^{\mu \nu}
$$

where the SU(3) couplings are given by the usual traces in flavor space, analogously to those in Eq. (C15).

An axial vector couples to octet baryons via

$$
\mathcal{L}_{A B B}=\bar{B}\left(g_{A B B}^{v} \gamma^{\mu}+g_{A B B}^{t} \frac{\mathrm{i} \sigma^{\mu \nu} q_{\nu}}{2 M_{S}}\right) \gamma_{5} A_{\mu} B
$$

The explicit expressions for the flavor couplings are analogous to those in Eq. (C15). Care must be taken in Eq. (C18) since only one of the couplings is allowed for a definite $G$-parity state of the axial meson $A$. Because of $G$ parity considerations, the vector coupling $g_{A B B}^{v}$ does not contribute to $b_{1}$ exchanges, and the tensor coupling $g_{A B B}^{t}$ is blocked for $a_{1}$ exchanges.

The Lagrangians that describe the octet-to-decuplet transitions via a vector-meson and a tensor-meson emission are $[102,103]$

$$
\begin{aligned}
\mathcal{L}_{\mathrm{VBD}}= & -\sqrt{3} \bar{D}_{i j k}^{\mu} \epsilon_{i l m}\left[-\mathrm{i} \frac{g_{\mathrm{VBD}}^{(1)}}{M_{S}} \gamma^{\nu} V_{\mu \nu, j l}-\frac{g_{\mathrm{VBD}}^{(2)}}{M_{S}^{2}} V_{\mu \nu, j l} \partial^{\nu}\right. \\
& \left.+\frac{g_{\mathrm{VBD}}^{(3)}}{M_{S}^{2}} \partial^{\nu} V_{\mu \nu, j l}\right] \gamma_{5} B_{k m}+\text { H.c., } \\
\mathcal{L}_{T B D}= & -\sqrt{3} \mathrm{i} \frac{g_{T B D}}{m_{T}} \bar{D}_{i j k}^{\lambda} \epsilon_{i l m}\left(g_{\lambda \mu} \partial_{\nu}+g_{\lambda \nu} \partial_{\mu}\right) \gamma_{5} B_{k m} T_{j l}^{\mu \nu} \\
& + \text { H.c. },
\end{aligned}
$$

where the explicit form of the fully symmetric decuplet matrix elements is 


$$
\begin{array}{rlrl}
T_{\mu}^{111} & =\Delta_{\mu}^{++}, & T_{\mu}^{112}=\frac{1}{\sqrt{3}} \Delta_{\mu}^{+}, & \\
T_{\mu}^{122}=\frac{1}{\sqrt{3}} \Delta_{\mu}^{0}, & T_{\mu}^{222}=\Delta_{\mu}^{-}, & \\
T_{\mu}^{113}=\frac{1}{\sqrt{3}} \Sigma_{\mu}^{*+}, & T_{\mu}^{123}=\frac{1}{\sqrt{6}} \Sigma_{\mu}^{* 0}, & T_{\mu}^{223}=\frac{1}{\sqrt{3}} \Sigma_{\mu}^{*-}, \\
T_{\mu}^{133}=\frac{1}{\sqrt{3}} \Xi_{\mu}^{* 0}, & T_{\mu}^{233}=\frac{1}{\sqrt{3}} \Xi_{\mu}^{*-}, & T_{\mu}^{333}=\Omega_{\mu}^{-} .
\end{array}
$$

Note that in the literature one often does not consider the minimal complete set of interaction Lagrangians when spin-3/2 baryons or tensor mesons are involved. In these analyses, one rather selects a single interaction term, which is usually unjustified. When considering $\hat{\beta}_{\mu_{V} \mu_{D}}^{\mathrm{VBD}}$ in Table VII, one observes that for $g_{\mathrm{VBD}}^{(2)}=g_{\mathrm{VBD}}^{(3)}=0$ and $m_{2}=m_{4}$, and neglecting $t$-dependent terms in $\hat{\beta}_{\mu_{V} \mu_{D}}^{\mathrm{VBD}}(t)$, we reproduce the quark-model results in [34]: $\beta_{-\frac{1}{2}+\frac{1}{2}}^{\mathrm{VBD}} / \beta_{+\frac{1}{2}+\frac{3}{2}}^{\mathrm{VBD}}=\sqrt{3}$ and $\beta_{+\frac{1}{2}+\frac{1}{2}}^{\mathrm{VBD}}=\beta_{-\frac{1}{2}+\frac{3}{2}}^{\mathrm{VBD}}=0$. Based on this correspondence, we set $g_{\mathrm{VBD}}^{(2)}=g_{\mathrm{VBD}}^{(3)}=0$ in the global SU(3)-EXD fit.

\section{APPENDIX D: ESTIMATING COUPLING CONSTANTS FROM DECAY RATIOS}

The coupling constants that appear in the meson Lagrangians can be estimated based on measured decay ratios. These couplings serve as starting values for the fits.

From the data on decay widths of tensor mesons into pseudoscalars, one can extract the numerical values for the couplings $c_{T P P}^{8}$ and $c_{T P P}^{1}$. When doing so in a global fit, Giacosa et al. [93] obtained ${ }^{9}$

$$
c_{T P P}^{8} \approx 11.7 \mathrm{GeV}^{-1}, \quad c_{T P P}^{1} \approx 13.6 \mathrm{GeV}^{-1} .
$$

The decay width of the $\rho$ into two pions is $\Gamma_{\rho \rightarrow \pi \pi}=$ $(149.1 \pm 0.8) \mathrm{MeV}$ [38]. Comparing this with the expression for the decay width in terms of the Lagrangian couplings, one finds $g_{\mathrm{VPP}}= \pm 4.2$. For completeness, it is worth mentioning that $g_{\mathrm{VPP}}= \pm 4.5$, when extracted from the $K^{*} \rightarrow K \pi$ decay width.

Estimating the coupling $g_{V V P}$ is less straightforward. The decay ratio of the $\phi$ meson into the $\omega \pi^{0}$ channel is consistent with 0 , both from theory and from experiment, while the only other channel measured so far is the decay of the $\phi$ into 3 pions, which can occur via the intermediate channel $\phi \rightarrow \rho \pi$. If one were to assume ideal mixing for the vector mesons, this coupling would vanish as well in the

\footnotetext{
${ }^{9}$ In [93], there was a typo in the results, which was solved in private communication; the values given in that paper are $c_{T P P}^{i, \text { Paper }}=\frac{\sqrt{2} F^{2}}{4} c_{T P P}^{i, \text { True }}$, where $F=92.4 \mathrm{MeV}$ is the pion decay constant.
}

OZI limit. Therefore, for this particular estimate, we use the vector-meson mixing angle $\theta_{V}=39^{\circ}$ [93]. Assuming that all 3-pion decays of the $\phi$ happen via the $\pi \rho$ intermediate channel, one then obtains $g_{V V P}=13.2 \mathrm{GeV}^{-1}$. Note that this is to be seen only as a starting value for the fits since for this particular value, many assumptions had to be made, which can give only a rough estimate of the coupling value.

To estimate $g_{T V P}$, we use the information that the decay $a_{2} \rightarrow 3 \pi$ with decay width $105 \mathrm{MeV}$ occurs dominantly through the $\rho \pi$ intermediate state. Assuming only this intermediate state, one finds a partial width of $73.5 \mathrm{MeV}$. This leads to $g_{T V P} \approx 6.8 \mathrm{GeV}^{-2}$. In fact, the coupling ranges between $6.4 \mathrm{GeV}^{-2}$ and $6.8 \mathrm{GeV}^{-2}$, when the decay fraction into a $\rho \pi$ intermediate state is varied between $88 \%$ and $100 \%$. This is consistent with the results from $K_{2}^{*} \rightarrow \pi K^{*}, K \rho$ and $K \omega$, where the estimated $g_{T V P}$ ranges between $6.4 \mathrm{GeV}^{-2}$ and $7.5 \mathrm{GeV}^{-2}$.

In order to estimate $g_{V P A}$, we use the total decay width $(142 \pm 9) \mathrm{MeV}$ of the $b_{1}$. It dominantly decays into $\omega \pi$, leading to $g_{V P A} \approx 4.0 \mathrm{GeV}$. All of the above-mentioned estimates are in good agreement with the quark-model predictions from [83].

One can extract the meson-baryon couplings using Eq. $(\mathrm{C} 15)$ and $(i=v, t)$

$$
\begin{gathered}
g_{\rho p p}^{i}=\frac{1}{\sqrt{2}}\left(g_{\mathrm{VBB}}^{i, F}+g_{\mathrm{VBB}}^{i, D}\right), \\
g_{\omega p p}^{i}=\frac{3 g_{\mathrm{VBB}}^{i, F}-g_{\mathrm{VBB}}^{i, D}}{3 \sqrt{2}}+\sqrt{\frac{2}{3}} g_{\mathrm{VBB}}^{i, S},
\end{gathered}
$$

and relating them to the empirical couplings from nucleonnucleon scattering data. The empirical results of the nucleon-nucleon Bonn potential from Refs. [104,105] read

$g_{\rho p p}^{v}=3.3, \quad g_{\omega p p}^{v}=16, \quad g_{\rho p p}^{t}=20, \quad g_{\omega p p}^{t}=0$.

Furthermore, from [106], one finds

$$
\frac{g_{v}^{F}}{g_{v}^{F}+g_{v}^{D}}=1, \quad \frac{g_{v}^{F}+g_{t}^{F}}{g_{v}^{F}+g_{v}^{D}+g_{t}^{F}+g_{t}^{D}}=\frac{2}{5} .
$$

Finally, one obtains

$$
\begin{aligned}
& g_{\mathrm{VBB}}^{v, F}=4.6, \quad g_{\mathrm{VBB}}^{v, D}=0, \quad g_{\mathrm{VBB}}^{v, S}=15.5, \\
& g_{\mathrm{VBB}}^{t, F}=8.4, \quad g_{\mathrm{VBB}}^{t, D}=19.6, \quad g_{\mathrm{VBB}}^{t, S}=-1.6 .
\end{aligned}
$$

The coupling $g_{T B B}^{(2)}$ in Eq. (C17) is often estimated to be compatible with 0 when tensor-meson dominance (TMD) is assumed. One might question the validity of the TMD approach. Indeed, in [97] the authors state that $g_{T B B}^{(1)} \approx-g_{T B B}^{(2)}$. 


\section{APPENDIX E: COVARIANT AND HELICITY AMPLITUDES}

From the interaction Lagrangians in Appendix C, one determines the helicity amplitudes by choosing the appropriate polarization angles. Since we consider high-energy scattering, the amplitudes are expanded in powers of $s$, and only the leading term is used. The $s$-channel helicity amplitudes are then cast onto the factorized form

$$
A_{\mu_{4} \mu_{3} \mu_{2} \mu_{1}}=\beta_{\mu_{1} \mu_{3}}^{e 13}(t) \beta_{\mu_{2} \mu_{4}}^{e 24}(t) \mathcal{P}_{e}(s, t)
$$

which coincides with the limit of our high-energy amplitudes in Eq. (6) for $t \rightarrow m_{e}^{2}$. We introduced the convenient notation for the meson propagator

$$
\mathcal{P}_{e} \equiv \mathcal{P}_{e}(s, t)=\frac{s^{J_{e}}}{m_{e}^{2}-t}
$$

From the analysis of kinematical singularities, we showed that the $s$-channel residues $\beta_{\mu_{i} \mu_{j}}^{e i j}(t)$ of an evasive reggeon must at least go as $\beta_{\mu_{i} \mu_{j}}^{e i j}(t) \sim \sqrt{-t}\left|\mu_{i}-\mu_{j}\right|$. In order to unambiguously factorize our amplitudes, we first consider $\pi \pi, \pi N$, and $N N$ scattering with a $t$-channel vector exchange $V$. One obtains the asymptotic expressions (up to isospin factors)

$$
\begin{gathered}
A_{0000}(\pi \pi \rightarrow \pi \pi)=2 g_{\mathrm{VPP}}^{2} \mathcal{P}_{V}, \\
A_{+0+0}(\pi N \rightarrow \pi N)=2 g_{\mathrm{VPP}} g_{\mathrm{VBB}}^{v} \mathcal{P}_{V}, \\
A_{++++}(N N \rightarrow N N)=2\left(g_{\mathrm{VBB}}^{v}\right)^{2} \mathcal{P}_{V}, \\
A_{+++-}(N N \rightarrow N N)=g_{v} \frac{g_{\mathrm{VBB}}^{t}}{M_{B}} \sqrt{-t^{\prime}} \mathcal{P}_{V} .
\end{gathered}
$$

From the above, we obtain

$$
\begin{gathered}
\beta_{00}^{\mathrm{VPP}}(t)=\sqrt{2} g_{\mathrm{VPP}}, \\
\beta_{++}^{\mathrm{VBB}}(t)=\sqrt{2} g_{\mathrm{VBB}}^{v}, \\
\beta_{-+}^{\mathrm{VBB}}(t)=\sqrt{2} \frac{g_{\mathrm{VBB}}^{t}}{m_{2}+m_{4}} \sqrt{-t^{\prime}} .
\end{gathered}
$$

A similar approach is followed for all reactions under consideration in this work. All residues considered in this work are listed in Table VII. The SU(3) flavor traces and commutators appear as factors then multiplying these residues.

As an example for how to build an amplitude inspired by single-particle-exchange, one can consider the helicity-flip contribution to the process $\pi^{-} p \rightarrow \pi^{0} n$. The full SU(3)constrained amplitude at high energies is given by

$$
\begin{aligned}
& A_{+0-0}\left(\pi^{-} p \rightarrow \pi^{0} n\right) \\
& \quad=e^{b_{\mathrm{sf}} t} \sqrt{-t} \hat{\beta}_{00}^{\rho \pi^{-} \pi^{0}}(t) \hat{\beta}_{-+}^{\rho p n}(t) \mathcal{F}_{\rho}(s, t) \mathcal{R}(s, t) .
\end{aligned}
$$

Here,

$$
\begin{gathered}
\hat{\beta}_{00}^{\rho \pi^{-} \pi^{0}}(t)=\sqrt{2}\left(\sqrt{2} g_{\mathrm{VPP}}\right), \\
\hat{\beta}_{-+}^{\rho p n}(t)=\frac{\sqrt{2}}{2 m_{N}}\left(g_{\mathrm{VBB}}^{t, F}+g_{\mathrm{VBB}}^{t, D}\right),
\end{gathered}
$$

where the factor in front of the parentheses is obtained from Table VII. The terms between parentheses are obtained by working out the flavor traces.

In the unconstrained fit, we have

$$
\begin{aligned}
& A_{+0-0}\left(\pi^{-} p \rightarrow \pi^{0} n\right) \\
& \quad=e^{\left(b^{\rho \pi \pi}+b_{-+}^{\rho N N}\right) t}\left(-g^{\rho \pi^{+} \pi^{+}}\right)\left(\sqrt{2} g_{-+}^{\rho p p}\right) \sqrt{-t} \mathcal{F}_{\rho}(s, t) \mathcal{R}(s, t)
\end{aligned}
$$

since we have made the arbitrary choice of fitting the coupling constants $g^{\rho \pi^{+} \pi^{+}}$and $\beta_{-+}^{\rho p p}$ and relating all other charge states to these couplings.

The differential cross section is computed using

$$
\frac{\mathrm{d} \sigma}{\mathrm{d} t}=\frac{1}{2 \times 16 \pi S_{12}^{2}(s)} \sum_{\mu_{i}}\left|A_{\mu_{4} \mu_{3} \mu_{2} \mu_{1}}\right|^{2} .
$$

\section{APPENDIX F: $t$-CHANNEL DECAY COUPLINGS}

In various single channel analyses, one starts from the $t$-channel to model the reggeon contributions. Hereby, $t$-channel helicity couplings (denoted by $\gamma_{\lambda_{i} \lambda_{k}}(t)$ ) are used. The $s$-channel residues can be related to the $t$-channel residues by analytically continuing the $s$-channel amplitudes to the $t$-channel. To distinguish between the helicity amplitudes in both channels, we explicitly mention the channel in a subscript. Additionally, we use $\mu$ and $\lambda$ for $s$ - and $t$-channel helicities, respectively.

Consider the $s$-channel amplitude for an exchange $e$ in Eq. (6). Near the lowest mass pole, we have the simple form for the $s$-channel amplitude

$$
A_{\mu_{4} \mu_{3} \mu_{2} \mu_{1}}^{s}(s, t)=\frac{\beta_{\mu_{2} \mu_{4}}(t) \beta_{\mu_{1} \mu_{3}}(t)}{m_{e}^{2}-t} s^{J_{e}} .
$$

Note that in the above, the residues include the $\sqrt{-t}$ factors, which must also be evaluated at $t=m_{e}^{2}$.

In the $t$-channel $a+\bar{c} \rightarrow \bar{b}+d$, the helicity amplitude can be expanded in partial waves through 
$A_{\lambda_{4} \lambda_{2} \lambda_{3} \lambda_{1}}^{t}(s, t)=16 \pi \sum_{J=M_{t}}(2 J+1) A_{\lambda_{4} \lambda_{2} \lambda_{3} \lambda_{1}, J}^{t}(t) d_{\lambda \lambda^{\prime}}^{J}\left(z_{t}\right)$,

where $\lambda=\lambda_{13}=\lambda_{1}-\lambda_{3}$ and $\lambda^{\prime}=\lambda_{24}=\lambda_{2}-\lambda_{4}$ and $M_{t}=\max \left\{|\lambda|,\left|\lambda^{\prime}\right|\right\}$. The $z_{t}$ is the cosine of the $t$-channel c.m. scattering angle. For a resonance $e$ with spin $J_{e}$, the corresponding partial-wave amplitude is parametrized as

$$
A_{\lambda_{4} \lambda_{2} \lambda_{3} \lambda_{1}, J_{e}}^{t}(t)=\frac{\gamma_{\lambda_{2} \lambda_{4}}(t) \gamma_{\lambda_{1} \lambda_{3}}(t)}{m_{e}^{2}-t} .
$$

In the following, we relate the $s$-channel residues $\beta$ in Eq. (F1) to the $t$-channel residues $\gamma$ in Eq. (F3), without invoking Lagrangians to carry out the crossing (which is the inverse direction of the derivation by Fox and Hey [33]).

We continue the $s$-channel helicity amplitudes into the $t$ channel and project them onto the $t$-channel helicity basis [107]

$$
\begin{aligned}
A_{\lambda_{4} \lambda_{2} \lambda_{3} \lambda_{1}}^{t}(s, t)= & -i \sum_{\mu_{i}} d_{\mu_{1} \lambda_{1}}^{s_{1}}\left(-\chi_{1}^{t \rightarrow s}\right) d_{\mu_{2} \lambda_{2}}^{s_{2}}\left(-\chi_{2}^{t \rightarrow s}\right) d_{\mu_{3} \lambda_{3}}^{s_{3}} \\
& \times\left(-\chi_{3}^{t \rightarrow s}\right) d_{\mu_{4} \lambda_{4}}^{s_{4}}\left(-\chi_{4}^{t \rightarrow s}\right) A_{\mu_{4} \mu_{3} \mu_{2} \mu_{1}}^{s}(s, t),
\end{aligned}
$$

where the $t \rightarrow s$-channel rotation angles are given by

$$
\begin{gathered}
\cos \chi_{i}^{t \rightarrow s}=\frac{(-1)^{c_{i}+1}\left(s+m_{i}^{2}-m_{j}^{2}\right)\left(t+m_{i}^{2}-m_{k}^{2}\right)-2 m_{i}^{2} \Delta_{m}}{S_{i j}(s) T_{i k}(t)}, \\
\sin \chi_{i}^{t \rightarrow s}=\frac{2 m_{i} \phi^{1 / 2}}{S_{i j}(s) T_{i k}(t)} \\
\Delta_{m}=m_{2}^{2}-m_{4}^{2}-m_{1}^{2}+m_{3}^{2}, \\
T_{i k}^{2}(t)=\left[t-\left(m_{i}+m_{k}\right)^{2}\right]\left[t-\left(m_{i}-m_{k}\right)^{2}\right] .
\end{gathered}
$$

Here, $\phi$ is the Kibble function, $c_{i}=1(=0)$ if particle $i$ is (not) crossed, $j$ is the $s$-channel, and $k$ the $t$-channel pair particle of $i$. Note that, following the path of Trueman and Wick [108], the square roots in the $s$-channel residues must be evaluated at $t=t-i \epsilon$ since we cross the real $s, t$ plane at negative $t$. This means that $\sqrt{-t}=i \sqrt{t}$. Note that we do not include a helicity dependent phase in Eq. (F4). This is in agreement with Trueman and Wick [108]. At leading $s$, we find that

$$
\begin{gathered}
S_{i j}(s)=s, \\
\sqrt{\phi}=s \sqrt{-t}, \\
\sin \chi_{i}^{t \rightarrow s}=\frac{2 m_{i} \sqrt{-t}}{T_{i k}(t)},
\end{gathered}
$$

$$
\cos \chi_{i}^{t \rightarrow s}=\frac{(-1)^{c_{i}+1}\left(t+m_{i}^{2}-m_{k}^{2}\right)}{T_{i k}(t)} .
$$

The rotation matrix has the property of being factorizable in a top-vertex and bottom-vertex rotation. If we assume for the moment that the left-hand side of Eq. (F4) can also be factorized

$$
A_{\lambda_{4} \lambda_{2} \lambda_{3} \lambda_{1}}^{t}(s, t)=A_{\lambda_{3} \lambda_{1}}^{t}(s, t) A_{\lambda_{4} \lambda_{2}}^{t}(s, t),
$$

then all of the above can be written in a factorized form

$A_{\lambda_{k} \lambda_{i}}^{t}(s, t)=\sum_{\mu_{i}, \mu_{k}} \sqrt{\mathcal{F}(s, t)} \beta_{\mu_{i} \mu_{k}}^{e i k}(t) d_{\mu_{i} \lambda_{i}}^{s_{i}}\left(-\chi_{i}^{t \rightarrow s}\right) d_{\mu_{k} \lambda_{k}}^{s_{k}}\left(-\chi_{k}^{t \rightarrow s}\right)$,

where $(i, k)=(1,3)$ or $(2,4)$. An additional factor of $i$ must be included for the fermion vertex.

In order to write Eq. (F2) in a factorized form, we realize that we are working in the high $s$ limit, where $z_{t}$ is large. The $d$-functions become factorizable when only their leading order in $z_{t}$ is considered

$$
\begin{aligned}
& A_{\lambda_{4} \lambda_{2} \lambda_{3} \lambda_{1}}^{t}(s, t) \\
& \quad=16 \pi \sum_{J=M_{t}}(2 J+1) A_{\lambda_{4} \lambda_{2} \lambda_{3} \lambda_{1}, J}^{t}(t) e^{-i \pi \lambda^{\prime}} d_{\lambda}^{J}\left(z_{t}\right) d_{\lambda^{\prime}}^{J}\left(z_{t}\right),
\end{aligned}
$$

where

$$
d_{\lambda}^{J}(z) \equiv e^{+i \pi \lambda / 2}\left[\left(\frac{z}{2}\right)^{J} \frac{\Gamma(2 J+1)}{\Gamma(J+|\lambda|+1) \Gamma(J-|\lambda|+1)}\right]^{1 / 2} .
$$

Note that the above form is not fully factorized in the sense that the $z_{t}$ depends on both top and bottom particle masses. We therefore consider the leading $s$ form of $z_{t}$ at constant $t$,

$$
z_{t}=\frac{2 t s}{T_{13}(t) T_{24}(t)},
$$

and introduce the functions

$$
\begin{aligned}
h_{\lambda_{i k}}^{J}\left(z_{t}\right) \equiv & e^{+i \pi \lambda_{i k} / 2}\left[\frac{\Gamma(2 J+1)}{\Gamma\left(J+\left|\lambda_{i k}\right|+1\right) \Gamma\left(J-\left|\lambda_{i k}\right|+1\right)}\right]^{1 / 2} \\
& \times\left(\frac{t s}{T_{i k}^{2}(t)}\right)^{J / 2} .
\end{aligned}
$$

Close to the $m_{e}$ pole in $t$, the partial-wave expansion is dominated by the $J_{e}$ partial wave. In Eq. (F3), we have assumed a factorizable form for the $t$-channel partial-wave amplitude. Therefore, we can rewrite the partial-wave amplitude as 


$$
A_{\lambda_{4} \lambda_{2} \lambda_{3} \lambda_{1}, J_{e}}^{t}(t)=A_{\lambda_{4} \lambda_{2}, J_{e}}^{t}(t) A_{\lambda_{3} \lambda_{1}, J_{e}}^{t}(t), \quad(\mathrm{F} 16) \quad \text { where }
$$

where

$$
A_{\lambda_{k} \lambda_{i}, J_{e}}^{t}(t)=\gamma_{\lambda_{i} \lambda_{k}}(t) \sqrt{\frac{1}{t-m_{e}^{2}}}
$$

$$
\xi\left(\lambda_{i k}\right)=e^{-i \pi \lambda_{i k}} \text { for a bottom vertex, else } \xi\left(\lambda_{i k}\right)=1 \text {. }
$$

The above can then be written in the factorized form

$$
A_{\lambda_{k} \lambda_{i}}^{t}(s, t)=\sqrt{16 \pi\left(2 J_{e}+1\right)} A_{\lambda_{k} \lambda_{i}, J_{e}}^{t}(t) h_{\lambda_{i k}}^{J_{e}}\left(z_{t}\right) \xi\left(\lambda_{i k}\right), \quad(\mathrm{F} 18)
$$

Putting everything together, we obtain the explicit form of the $t$-channel residue as a function of the $s$-channel residue

$$
\gamma_{\lambda_{i} \lambda_{k}}(t)=\frac{1}{\sqrt{16 \pi\left(2 J_{e}+1\right)}} \frac{s^{J_{e} / 2}}{\xi\left(\lambda_{i k}\right) h_{\lambda_{i k}}^{J_{e}}\left(z_{t}\right)} \sum_{\mu_{i}, \mu_{k}} \beta_{\mu_{i} \mu_{k}}^{e i k}(t) d_{\mu_{i} \lambda_{i}}^{s_{i}}\left(-\chi_{i}^{t \rightarrow s}\right) d_{\mu_{k} \lambda_{k}}^{s_{k}}\left(-\chi_{k}^{t \rightarrow s}\right) .
$$

Note that the above is only valid for $s \rightarrow \infty$ and $t \rightarrow m_{e}^{2}$. In order to explicitly illustrate the cancellation of the $s$ dependence, we focus on the $h_{\lambda_{i k}}^{J_{e}}\left(z_{t}\right)$ function. Using Eq. (F15), one obtains

$$
\frac{h_{\lambda_{e}}^{J_{e}}\left(z_{t}\right)}{s^{J} J / 2}=e^{+i \lambda_{i k} / 2}\left(\frac{t}{T_{i k}^{2}(t)}\right)^{J_{e} / 2}\left[\frac{\Gamma\left(2 J_{e}+1\right)}{\Gamma\left(J_{e}+\left|\lambda_{i k}\right|+1\right) \Gamma\left(J_{e}-\left|\lambda_{i k}\right|+1\right)}\right]^{1 / 2} .
$$

Hence (and being more precise in the notation)

$$
\begin{aligned}
\gamma_{\lambda_{1} \lambda_{3}}^{e}\left(t=m_{e}^{2}\right)= & \frac{e^{-i \pi \lambda_{13} / 2}}{\sqrt{16 \pi\left(2 J_{e}+1\right)}}\left(\frac{T_{13}\left(m_{e}^{2}\right)}{m_{e}}\right)^{J_{e}}\left[\frac{\Gamma\left(J_{e}+\left|\lambda_{13}\right|+1\right) \Gamma\left(J_{e}-\left|\lambda_{13}\right|+1\right)}{\Gamma\left(2 J_{e}+1\right)}\right]^{1 / 2} \\
& \times \sum_{\mu_{1}, \mu_{3}} \beta_{\mu_{1} \mu_{3}}\left(t=m_{e}^{2}\right) d_{\mu_{1} \lambda_{1}}^{s_{1}}\left(-\left.\chi_{1}^{t \rightarrow s}\right|_{t=m_{e}^{2}}\right) d_{\mu_{3} \lambda_{3}}^{s_{3}}\left(-\left.\chi_{3}^{t \rightarrow s}\right|_{t=m_{e}^{2}}\right)
\end{aligned}
$$

for the top vertex and

$$
\begin{aligned}
\gamma_{\lambda_{2} \lambda_{4}}^{e}\left(t=m_{e}^{2}\right)= & \frac{(-i) e^{+i \pi \lambda_{24} / 2}}{\sqrt{16 \pi\left(2 J_{e}+1\right)}}\left(\frac{T_{24}\left(m_{e}^{2}\right)}{m_{e}}\right)^{J_{e}}\left[\frac{\Gamma\left(J_{e}+\left|\lambda_{24}\right|+1\right) \Gamma\left(J_{e}-\left|\lambda_{24}\right|+1\right)}{\Gamma\left(2 J_{e}+1\right)}\right]^{1 / 2} \\
& \times \sum_{\mu_{2}, \mu_{4}} \beta_{\mu_{2} \mu_{4}}\left(t=m_{e}^{2}\right) d_{\mu_{2} \lambda_{2}}^{s_{2}}\left(-\left.\chi_{2}^{t \rightarrow s}\right|_{t=m_{e}^{2}}\right) d_{\mu_{4} \lambda_{4}}^{s_{4}}\left(-\left.\chi_{4}^{t \rightarrow s}\right|_{t=m_{e}^{2}}\right)
\end{aligned}
$$

for the bottom vertex. The crossing angles must be evaluated at the pole

$$
\begin{gathered}
\left.\sin \chi_{i}\right|_{t=m_{e}^{2}}=i \frac{2 m_{i} m_{e}}{\lambda^{1 / 2}\left(m_{e}^{2}, m_{i}^{2}, m_{k}^{2}\right)}, \\
\left.\cos \chi_{i}\right|_{t=m_{e}^{2}}=\frac{(-1)^{c_{i}+1}\left(m_{e}^{2}+m_{i}^{2}-m_{k}^{2}\right)}{\lambda^{1 / 2}\left(m_{e}^{2}, m_{i}^{2}, m_{k}^{2}\right)} .
\end{gathered}
$$

In summary, Eqs. (F22) and (F23) relate the $s$-channel residues at the pole directly to the $t$-channel residues. With these expressions, one can compare the results obtained in this work directly to the decay couplings of various processes. 
[1] M. Battaglieri et al., Acta Phys. Polon. B 46, 257 (2015).

[2] K. Goeke, M. V. Polyakov, and M. Vanderhaeghen, Prog. Part. Nucl. Phys. 47, 401 (2001).

[3] J. T. Londergan, J. Nebreda, J. R. Pel aez, and A. Szczepaniak, Phys. Lett. B 729, 9 (2014).

[4] C. Fernández-Ramírez, I. V. Danilkin, V. Mathieu, and A. P. Szczepaniak, Phys. Rev. D 93, 074015 (2016).

[5] J. R. Peláez and A. Rodas, Eur. Phys. J. C 77, 431 (2017).

[6] J. Mandula, J. Weyers, and G. Zweig, Ann. Rev. Nucl. Part. Sci. 20, 289 (1970).

[7] H. Al Ghoul et al. (GlueX Collaboration), Phys. Rev. C 95, 042201 (2017).

[8] D. I. Glazier, Acta Phys. Pol. B Proc. Suppl. 8, 503 (2015).

[9] P. D. B. Collins, An Introduction to Regge Theory and High-Energy Physics, Cambridge Monographs on Mathematical Physics (Cambridge Univ. Press, Cambridge, UK, 2009).

[10] V. Mathieu, I. V. Danilkin, C. Fernández-Ramírez, M. R. Pennington, D. Schott, A. P. Szczepaniak, and G. Fox, Phys. Rev. D 92, 074004 (2015).

[11] V. Mathieu, G. Fox, and A. P. Szczepaniak, Phys. Rev. D 92, 074013 (2015).

[12] J. Nys, V. Mathieu, C. Fernández-Ramírez, A. N. Hiller Blin, A. Jackura, M. Mikhasenko, A. Pilloni, A. P. Szczepaniak, G. Fox, and J. Ryckebusch (JPAC Collaboration), Phys. Rev. D 95, 034014 (2017).

[13] J. Nys, V. Mathieu, C. Fernández-Ramírez, A. Jackura, M. Mikhasenko, A. Pilloni, N. Sherrill, J. Ryckebusch, A. P. Szczepaniak, and G. Fox (JPAC Collaboration), Phys. Lett. B 779, 77 (2018).

[14] V. Mathieu, J. Nys, A. Pilloni, C. Fernández-Ramírez, A. Jackura, M. Mikhasenko, V. Pauk, A. Szczepaniak, and G. Fox (JPAC Collaboration), Europhys. Lett. 122, 41001 (2018).

[15] V. Mathieu, J. Nys, C. Fernández-Ramírez, A. Jackura, A. Pilloni, N. Sherrill, A. P. Szczepaniak, and G. Fox (JPAC Collaboration), Phys. Rev. D 97, 094003 (2018).

[16] V. Mathieu, J. Nys, C. Fernández-Ramírez, A. Jackura, M. Mikhasenko, A. Pilloni, A. P. Szczepaniak, and G. Fox, Phys. Lett. B 774, 362 (2017).

[17] H. Kamano, S. X. Nakamura, T. S. H. Lee, and T. Sato, Phys. Rev. C 88, 035209 (2013).

[18] A. V. Anisovich, R. Beck, E. Klempt, V. A. Nikonov, A. V. Sarantsev, and U. Thoma, Eur. Phys. J. A 48, 15 (2012).

[19] A. V. Anisovich, R. Beck, E. Klempt, V. A. Nikonov, A. V. Sarantsev, and U. Thoma, Eur. Phys. J. A 48, 88 (2012).

[20] D. Rönchen, M. Döring, F. Huang, H. Haberzettl, J. Haidenbauer, C. Hanhart, S. Krewald, U. G. Meißner, and K. Nakayama, Eur. Phys. J. A 50, 101 (2014); 51, 63(E) (2015).

[21] D. Rönchen, M. Döring, H. Haberzettl, J. Haidenbauer, U. G. Meißner, and K. Nakayama, Eur. Phys. J. A 51, 70 (2015).

[22] W.-T. Chiang, S.-N. Yang, L. Tiator, and D. Drechsel, Nucl. Phys. A700, 429 (2002).

[23] R. L. Workman, M. W. Paris, W. J. Briscoe, and I. I. Strakovsky, Phys. Rev. C 86, 015202 (2012).

[24] W. J. Briscoe, A. E. Kudryavtsev, P. Pedroni, I. I. Strakovsky, V. E. Tarasov, and R. L. Workman, Phys. Rev. C 86, 065207 (2012).
[25] M. C. Kunkel et al. (CLAS Collaboration), Phys. Rev. C 98, 015207 (2018).

[26] T. Beattie, Z. Papandreou, and J. Stevens (GlueX Collaboration), APS Division of Nuclear Physics Meeting, October 2017.

[27] G. C. Fox and E. Leader, Phys. Rev. Lett. 18, 628 (1967).

[28] G. C. Fox, ANL/HEP-7208, Vol. II, 1970.

[29] V. Mathieu, J. Nys, C. Fernández-Ramírez, A. N. Hiller Blin, A. Jackura, A. Pilloni, A. P. Szczepaniak, and G. Fox (JPAC Collaboration), Phys. Rev. D 98, 014041 (2018).

[30] G. Cohen-Tannoudji, P. Salin, and A. Morel, Nuovo Cimento A 55, 412 (1968).

[31] V. N. Gribov, Strong Interactions of Hadrons at High Energies: Gribov Lectures on Theoretical Physics, edited by Y.L. Dokshitzer and J. Nyiri (Cambridge University Press, Cambridge, UK, 2012).

[32] H. Harari, Phys. Rev. Lett. 22, 562 (1969).

[33] G. C. Fox and A. J. G. Hey, Nucl. Phys. B56, 386 (1973).

[34] A. C. Irving and R. P. Worden, Phys. Rep. 34, 117 (1977).

[35] M. Mikhasenko, A. Pilloni, J. Nys, M. Albaladejo, C. Fernández-Ramírez, A. Jackura, V. Mathieu, N. Sherrill, T. Skwarnicki, and A. P. Szczepaniak (JPAC Collaboration), Eur. Phys. J. C 78, 229 (2018).

[36] A. Pilloni, J. Nys, M. Mikhasenko, M. Albaladejo, C. Fernández-Ramírez, A. Jackura, V. Mathieu, N. Sherrill, T. Skwarnicki, and A. P. Szczepaniak (JPAC Collaboration), arXiv:1805.02113.

[37] A. C. Irving and C. Michael, Nucl. Phys. B82, 282 (1974).

[38] C. Patrignani et al. (Particle Data Group Collaboration), Chin. Phys. C 40, 100001 (2016).

[39] A. V. Barnes, D. J. Mellema, A. V. Tollestrup, R. L. Walker, O. I. Dahl, R. A. Johnson, R. W. Kenney, and M. Pripstein, Phys. Rev. Lett. 37, 76 (1976).

[40] A. V. Stirling et al., Phys. Rev. Lett. 14, 763 (1965).

[41] I. J. Bloodworth, W. C. Jackson, H. Merdjanian, J. D. Prentice, and T. S. Yoon, Nucl. Phys. B81, 231 (1974).

[42] R. Honecker et al. (Aachen-Berlin-Bonn-CERN-CracowHeidelberg Collaboration), Nucl. Phys. B131, 189 (1977).

[43] D. J. Schotanus, C. L. Pols, D. Z. Toet, R. T. Van De Walle, J. V. Major, G. E. Pearson, B. Chaurand, R. Vanderhaghen, G. Rinaudo, and A. E. Werbrouck (Durham-NijmegenParis-Torino Collaboration), Nucl. Phys. B22, 45 (1970).

[44] J. H. Scharenguivel, L. J. Gutay, J. A. Gaidos, S. L. Kramer, S. Lichtman, D. H. Miller, and K. V. Vasavada, Nucl. Phys. B36, 363 (1972).

[45] W. D. Apel et al. (Serpukhov-CERN Collaboration), Nucl. Phys. B152, 1 (1979); Sov. J. Nucl. Phys. 29, 780 (1979).

[46] V. N. Bolotov, V. V. Isakov, D. B. Kakauridze, V. A. Kachanov, V. M. Kutin, Yu. D. Prokoshkin, E. A. Rasuvaev, and V. K. Semenov, Yad. Fiz. 18, 1262 (1973).

[47] O. I. Dahl, R. A. Johnson, R. W. Kenney, M. Pripstein, A. V. Barnes, D. J. Mellema, A. V. Tollestrup, and R. L. Walker, Phys. Rev. Lett. 37, 80 (1976).

[48] C. Daum et al. (ACCMOR Collaboration), Zeitschrift für Physik C Particles and Fields 8, 95 (1981).

[49] W. D. Apel et al. (Serpukhov-CERN Collaboration), Phys. Lett. B 83, 131 (1979); [Sov. J. Nucl. Phys. 30, 189 (1979)].

[50] R. Diebold, D. S. Ayres, A. F. Greene, S. L. Kramer, A. J. Pawlicki, and A. B. Wicklund, Phys. Rev. Lett. 32, 904 (1974). 
[51] M. G. D. Gilchriese et al., Phys. Rev. Lett. 40, 6 (1978).

[52] J. J. Phelan, I. Ambats, W. T. Meyer, B. Musgrave, J. Rest, C. E. W. Ward, and H. Yuta, Phys. Lett. B 61, 483 (1976).

[53] I. Ambats, A. Lesnik, W. T. Meyer, D. R. Rust, C. E. W. Ward, and D. D. Yovanovitch, Nucl. Phys. B77, 269 (1974).

[54] P. Astbury et al., Phys. Lett. 16, 328 (1965).

[55] F. G. Binon et al. (Serpukhov-Brussels-Annecy(LAPP) Collaboration), Nuovo Cimento A 64, 89 (1981).

[56] V. N. Bolotov, V. V. Isakov, D. B. Kakauridze, V. A. Kachanov, V. M. Kutin, V. E. Postoev, Yu. D. Prokoshkin, and V. K. Semenov, Yad. Fiz. 18, 1061 (1973).

[57] G. W. Brandenburg, R. K. Carnegie, R. J. Cashmore, M. Davier, D. W. G. S. Leith, J. A. J. Matthews, W. T. Meyer, P. Walden, and S.H. Williams, Phys. Rev. D 15, 617 (1977).

[58] B. Chaurand et al., Nucl. Phys. B117, 1 (1976).

[59] J. Gallivan et al., Nucl. Phys. B117, 269 (1976).

[60] J. N. Carney et al., Nucl. Phys. B107, 381 (1976).

[61] K. J. Foley, W. A. Love, S. Ozaki, E. D. Platner, A. C. Saulys, E. H. Willen, S. Lindenbaum, and M. A. Kramer, Phys. Rev. D 9, 42 (1974).

[62] P. A. Baker, J. S. Chima, P. J. Dornan, D. J. Gibbs, G. Hall, D. B. Miller, T. S. Virdee, and A. P. White, Nucl. Phys. B166, 207 (1980).

[63] J. Ballam et al., Phys. Rev. Lett. 41, 676 (1978).

[64] M. Baubillier et al. (Birmingham-CERN-GlasgowMichigan State-Paris Collaboration), Z. Phys. C 23, 213 (1984).

[65] A. Berglund et al., Phys. Lett. B 73, 369 (1978).

[66] S. Al-Harran et al., Nucl. Phys. B183, 269 (1981).

[67] A. Bashian, G. Finocchiaro, M. L. Good, P. D. Grannis, O. Guisan, J. Kirz, Y. Y. Lee, R. Pittman, G. C. Fischer, and D. D. Reeder, Phys. Rev. D 4, 2667 (1971).

[68] G. S. Bitsadze et al., Nucl. Phys. B260, 497 (1985).

[69] D. J. Crennell, H. A. Gordon, K.-W. Lai, and J. M. Scarr, Phys. Rev. D 6, 1220 (1972).

[70] K. J. Foley, W. A. Love, S. Ozaki, E. D. Platner, A. C. Saulys, E. H. Willen, and S. J. Lindenbaum, Phys. Rev. D 8, 27 (1973).

[71] C. E. W. Ward, I. Ambats, A. Lesnik, W. T. Meyer, D. R. Rust, and D. D. Yovanovitch, Phys. Rev. Lett. 31, 1149 (1973).

[72] W. D. Apel et al. (Serpukhov-CERN Collaboration), Yad. Fiz. 31, 167 (1980); Lett. Nuovo Cimento 25, 493 (1979).

[73] O. I. Dahl, R. A. Johnson, R. W. Kenney, M. Pripstein, A. V. Barnes, D. J. Mellema, A. V. Tollestrup, and R. L. Walker, Phys. Rev. Lett. 38, 54 (1977).

[74] M. H. Shaevitz et al. (Carleton-Michigan State-Ohio StateToronto Collaboration), Phys. Rev. Lett. 36, 8 (1976).

[75] K. Paler, J. Tebes, R. C. Badewitz, H. R. Barton, Jr., D. H. Miller, and T. R. Palfrey, Jr., Lett. Nuovo Cimento 4, 745 (1972).

[76] A. P. Szczepaniak and M. R. Pennington, Phys. Lett. B 737, 283 (2014).

[77] T. Feldmann, P. Kroll, and B. Stech, Phys. Rev. D 58, 114006 (1998).
[78] J. L. Goity, A. M. Bernstein, and B. R. Holstein, Phys. Rev. D 66, 076014 (2002).

[79] B. Aubert et al. (BABAR Collaboration), Phys. Rev. D 74, 012002 (2006).

[80] V. Mathieu and V. Vento, Phys. Lett. B 688, 314 (2010).

[81] R. Escribano, P. Masjuan, and P. Sanchez-Puertas, Eur. Phys. J. C 75, 414 (2015).

[82] A. A. Osipov, B. Hiller, and A. H. Blin, Phys. Rev. D 93, 116005 (2016).

[83] S. Godfrey and N. Isgur, Phys. Rev. D 32, 189 (1985).

[84] J. J. Dudek, R. G. Edwards, P. Guo, and C. E. Thomas (Hadron Spectrum Collaboration), Phys. Rev. D 88, 094505 (2013).

[85] A. V. Anisovich, C. A. Baker, C. J. Batty, D. V. Bugg, L. Montanet, V. A. Nikonov, A. V. Sarantsev, V. V. Sarantsev, and B. S. Zou, Phys. Lett. B 542, 8 (2002).

[86] A. V. Anisovich, C. A. Baker, C. J. Batty, D. V. Bugg, L. Montanet, V. A. Nikonov, A. V. Sarantsev, V. V. Sarantsev, and B. S. Zou, Phys. Lett. B 542, 19 (2002).

[87] JPAC Collaboration, http://www.indiana.edu/ jpac/.

[88] V. Mathieu, AIP Conf. Proc. 1735, 070004 (2016).

[89] M. Jacob and G. C. Wick, Ann. Phys. (N.Y.) 7, 404 (1959); 281, 774 (2000).

[90] P. H. Frampton, Nucl. Phys. B7, 507 (1968).

[91] S. Bellucci, J. Gasser, and M. E. Sainio, Nucl. Phys. B423, 80 (1994); B431, 413(E) (1994).

[92] C.-K. Chow and S.-J. Rey, J. High Energy Phys. 05 (1998) 010.

[93] F. Giacosa, T. Gutsche, V. E. Lyubovitskij, and A. Faessler, Phys. Rev. D 72, 114021 (2005).

[94] L. Roca, E. Oset, and J. Singh, Phys. Rev. D 72, 014002 (2005).

[95] L. Roca, A. Hosaka, and E. Oset, Phys. Lett. B 658, 17 (2007).

[96] D. Drechsel, O. Hanstein, S. S. Kamalov, and L. Tiator, Nucl. Phys. A645, 145 (1999).

[97] H. Kleinert and P. H. Weisz, Lett. Nuovo Cimento 2, 459 (1971).

[98] B. G. Yu, T. K. Choi, and W. Kim, Phys. Rev. C 83, 025208 (2011).

[99] Y.-s. Oh and T. S. H. Lee, Phys. Rev. C 69, 025201 (2004).

[100] B. G. Yu, T. K. Choi, and W. Kim, Phys. Lett. B 701, 332 (2011).

[101] Y. Huang, J.-j. Xie, J. He, X. Chen, and H.-f. Zhang, arXiv:1606.04623.

[102] S.-i. Nam and B.-G. Yu, Phys. Rev. C 84, 025203 (2011).

[103] B.-G. Yu and K.-J. Kong, Phys. Lett. B 769, 262 (2017).

[104] R. Machleidt, K. Holinde, and C. Elster, Phys. Rep. 149, 1 (1987).

[105] R. Machleidt, Phys. Rev. C 63, 024001 (2001).

[106] C. B. Dover and A. Gal, Prog. Part. Nucl. Phys. 12, 171 (1985).

[107] A. Martin and T. Spearman, Elementary Particle Theory (North-Holland, Amsterdam, 1970).

[108] T. L. Trueman and G. C. Wick, Ann. Phys. (N.Y.) 26, 322 (1964). 